\title{
BMJ Open Systematic review of the factors and the key indicators that identify doctors at risk of complaints, malpractice claims or impaired performance
}

\author{
Elizabeth E Austin (D) , ${ }^{1}$ Vu Do, ${ }^{1}$ Ruqaiya Nullwala, ${ }^{1}$ Diana Fajardo Pulido, ${ }^{1}$ \\ Peter D Hibbert (D) , ' Jeffrey Braithwaite (D) , ${ }^{1}$ Gaston Arnolda (D) ,1 \\ Louise K Wiles, ${ }^{1,2}$ Tahlia Theodorou, ${ }^{1}$ Yvonne Tran, ${ }^{1}$ Reidar P Lystad (1) ,1 \\ Sarah Hatem, ${ }^{1}$ Janet C Long (D) , ${ }^{1}$ Frances Rapport (D) , ${ }^{1}$ Annette Pantle, ${ }^{3}$ \\ Robyn Clay-Williams (1) ${ }^{1}$
}

To cite: Austin EE, Do V, Nullwala $\mathrm{R}$, et al. Systematic review of the factors and the key indicators that identify doctors at risk of complaints, malpractice claims or impaired performance. BMJ Open 2021;11:e050377. doi:10.1136/ bmjopen-2021-050377

- Prepublication history and additional supplemental material for this paper are available online. To view these files, please visit the journal online. (http://dx.doi.org/10.1136/ bmjopen-2021-050377)

Received 18 February 2021 Accepted 05 August 2021

Check for updates

(C) Author(s) (or their employer(s)) 2021. Re-use permitted under CC BY-NC. No commercial re-use. See rights and permissions. Published by BMJ.

${ }^{1}$ Australian Institute of Health Innovation, Macquarie University, Sydney, New South Wales, Australia

${ }^{2}$ Australian Centre for Precision Health, University of South Australia, Adelaide, South Australia, Australia

${ }^{3}$ Medical Council of New South Wales, Sydney, New South Wales, Australia

Correspondence to Dr Elizabeth E Austin; elizabeth.austin@mq.edu.au

\section{ABSTRACT}

Objective To identify the risk factors associated with complaints, malpractice claims and impaired performance in medical practitioners.

Design Systematic review.

Data sources Ovid-Medline, Ovid Embase, Scopus and Cochrane Central Register of Controlled Trials were searched from 2011 until March 2020. Reference lists and Google were also handsearched.

Results Sixty-seven peer-reviewed papers and three grey literature publications from 2011 to March 2020 were reviewed by pairs of independent reviewers. Twentythree key factors identified, which were categorised as demographic or workplace related. Gender, age, years spent in practice and greater number of patient lists were associated with higher risk of malpractice claim or complaint. Risk factors associated with physician impaired performance included substance abuse and burn-out.

Conclusions It is likely that risk factors are interdependent with no single factor as a strong predictor of a doctor's risk to the public. Risk factors for malpractice claim or complaint are likely to be country specific due to differences in governance structures, processes and funding. Risk factors for impaired performance are likely to be specialty specific due to differences in work culture and access to substances. New ways of supporting doctors might be developed, using risk factor data to reduce adverse events and patient harm.

PROSPERO registration number PROSPERO registration number: CRD42020182045.

Medical practitioners have a responsibility to ensure the delivery of high quality and safe patient care. At times, the care provided may not be considered satisfactory or result in poor patient outcomes and a complaint may be lodged against a practitioner. Practitioners may receive complaints directly from patients, or through employers or organisations that do not have regulatory or licensing powers. Complaints about a clinician's conduct can
Strengths and limitations of this study

- Comprehensive search terminology and rigorous review methodology to identify studies from a range of academic databases and grey literature sources.

- A large number of factors associated with doctors at risk of malpractice claims, complaints or impaired performance were identified.

- High levels of heterogeneity precluded conducting pooled analyses.

- Differences in healthcare culture and governance between countries may limit the generalisability of the findings.

also be lodged with practitioner licensing boards. ${ }^{1}$ Some practitioner licensing boards carry out investigations into practitioner conduct, largely relying on complaints and claims from patients, peers and employers to identify potential instances of misconduct and impaired performance (ie, impairment in ability to practice). ${ }^{2}$ In contrast, claims are lawsuits or statements that have been filed for compensation for injuries caused by alleged negligence or omission. ${ }^{3}{ }^{4}$ However, not all impaired performance results in, or justifies a complaint or claim. Impaired performance, therefore, may provide insights into quality and safety-related problems before they result in poor patient outcomes and subsequent formal complaints or malpractice claims. Factors that interfere with a doctor's ability to function pose a risk to patient safety, whether they result in complaints, claims or impaired performance.

The identification of practitioners at higher risk of complaints and subsequent claims has been examined in previous studies ${ }^{5-7}$ Several predictive factors have been identified, 
and these can be categorised as system or personal risk factors. Examples of system factors include country of initial training, clinical workload and practice setting or sector (eg, solo vs group practice, private vs public health sector) ${ }^{8}$ Personal, or 'demographic', risk factors are specific to the individual; characteristics that have been reported to affect risk of complaints include age, sex, mental state, medical specialty and number of prior complaints. ${ }^{7}$ Given the increasing volume and complexity of patient care needs, as well as technical changes in clinical practice, it is necessary to gain a better insight into the factors which may lead to complaints, malpractice claims or impaired performance.

Understanding the factors contributing to risk will allow practitioner boards to make more objective assessments of doctors on receipt of a complaint and allow for better targeted monitoring of higher risk medical practitioners with imposed conditions or restrictions on their registration. ${ }^{9}$ Therefore, the purpose of this systematic review was to examine the research evidence provided in peer-reviewed and grey literature, to identify the risk factors associated with complaints, malpractice claims and impaired performance in medical practitioners.

\section{METHODS}

The published protocol (Prospero registration number: CRD42020182045) guided the review in accordance with the Preferred Reporting Items for Systematic Reviews and Meta-Analyses statement (PRISMA). ${ }^{10}$

\section{Search strategy}

A comprehensive search strategy was developed in consultation with a research librarian to search Ovid-Medline, Ovid Embase, Scopus and the Cochrane Central Register of Controlled Trials for peer-reviewed literature and Google for grey literature. Databases were searched on 27 March 2020 for English language articles published between 2011 and 2020. This was accompanied by handsearching the reference lists of relevant review articles. The full search strategy for all databases is shown in online supplemental appendix A.

An example, illustrating the search strategy for Scopus, is as follows:

TITLE-ABS-KEY("Doctor*" OR "physician*" OR "medical officer*") AND TITLE-ABSKEY ("malpractice" OR "negligen*" OR "impair*") AND TITLE-ABS-KEY("risk*")

\section{Eligibility criteria}

Original peer-reviewed research studies and grey literature articles were included in the systematic review if they met the following additional criteria: (1) involving doctors; (2) identifying risk factors for impaired performance and malpractice claims or complaint; (3) providing disaggregated demographic characteristics about the doctors in the sample. For this review, the terms 'physician', 'doctor' and 'medical practitioner' are used interchangeably to refer to registered medical doctors. Articles were excluded if they were: (1) systematic reviews, reviews of reviews (umbrella reviews) and other research syntheses; (2) grey literature opinion, letters to the editor, commentary or case report; involved student doctors or patients; (3) reported aggregated sample characteristics; (4) published before 2011; (5) published in a language other than English and (6) focused on system breakdown factors (eg, informed consent processes, organisational use of medical device or treatment, management processes) defensive medicine or litigation.

\section{Screening and data extraction}

The results of the searches were entered into EndNote citation management software (V.8.2; Thompson Reuters, New York, New York, USA), and duplicates were removed. The study titles and abstracts were entered into Rayyan, a free web and mobile application screening tool. ${ }^{11}$ For each study, title and abstract were independently screened by pairs of reviewers for inclusion according to the prespecified criteria. Disagreements were resolved via discussion. Abstracts flagged as potentially relevant by reviewers underwent full-text review, again by independent pairs of reviewers.

The data were extracted independently by pairs of reviewers into a form specifically designed for the review and piloted for usability prior to data extraction. The extraction form included author(s) name, year of publication, country where the study was conducted, study design, characteristics and risk factors of doctors and related data (eg, measures of relative risk such as OR, rate ratios, HRs), study limitations and study results. Where disaggregated data were reported for multiple professions, only data reported for doctors were extracted.

\section{Risk of bias}

Methodological quality of the included peer-reviewed studies was assessed using the following The Joanna Briggs Institute critical appraisal tools: Checklist for Cohort Studies, Checklist for Analytical Cross-Sectional Studies, Checklist for Randomised Controlled Trials, Checklist for Quasi-Experimental Studies and the Checklist for CaseControl Studies. ${ }^{12} 13$ Tools were selected based on study design and piloted on a sample of six research papers. Study quality was appraised by pairs of independent reviewers, with disagreements resolved via discussion.

\section{Data processing and analysis}

A narrative synthesis was performed for this review. Synthesis included numerical statistical summaries, textual commentaries, and tabular and graphical representations.

\section{Patient and public involvement}

Patients and the public were not involved in the design and conduct of this review. 


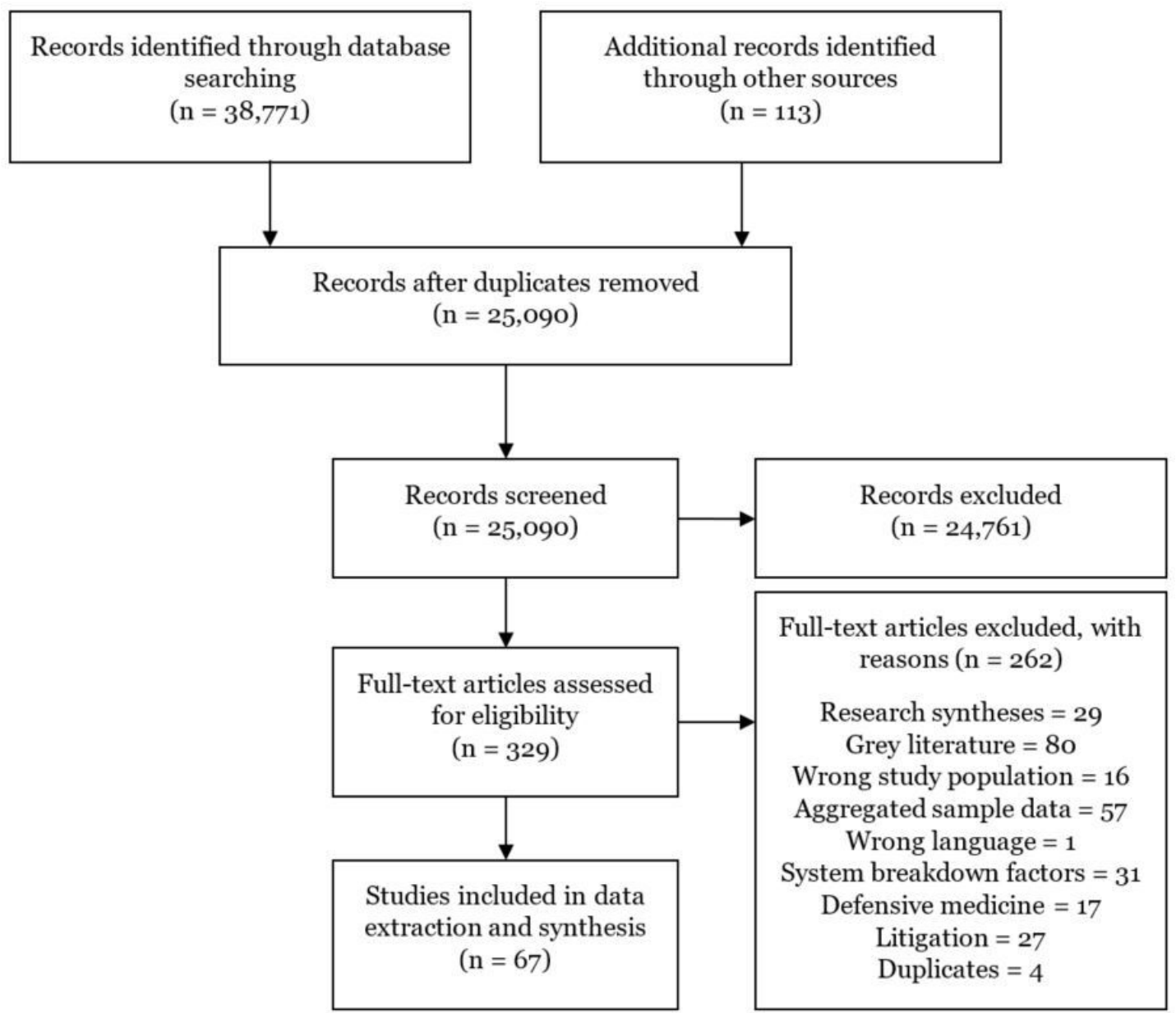

Figure 1 PRISMA flow diagram for study selection. PRISMA, Preferred Reporting Items for Systematic Reviews and MetaAnalyses statement.

\section{RESULTS}

\section{Literature search}

The combined searches yielded 38884 articles. Following removal of 13794 duplicates, 25090 abstracts and 329 fulltexts were reviewed, with 67 articles meeting inclusion criteria. Figure 1 presents the PRISMA diagram for the identification, screening, and inclusion processes. The grey literature search identified 11 reports, with three meeting the inclusion criteria.

\section{Description of the included studies}

Characteristics of the included studies are in table 1 . Publication years of included studies (2011-2020) are provided in figure 2. Most studies were conducted in the USA $(60 \%, n=40)$, followed by Australia $(12 \%, n=8)$. Two studies were conducted each in Canada, Denmark, Japan, Taiwan and the UK. One study was conducted in each of Chile, Columbia, Egypt, France, India, Iran, Italy, The Netherlands and Spain.

\section{Quality assessment}

Most studies were assessed as having considerable or potential flaws or limitations in their design, conduct or analysis that could distort the results (see table 2).

\section{Key factors}

The included 67 articles identified 23 factors associated with doctors at risk of malpractice claims, complaints and/or impaired performance. The 23 factors, categorised as either demographic or workplace related, are shown in figure 3 . Due to the variety of different settings and methods, it was deemed that the methodological heterogeneity was too broad to permit the calculation of pooled results. The results from studies are therefore reported in the form of a narrative synthesis.

\section{Demographic characteristics}

Age

\section{Complaints}

Older doctors were found to be at 1.4 times greater risk of complaints when compared with younger doctors. ${ }^{9}$ One study found older doctors (aged 61-70 years) to have the lowest complaint rates. ${ }^{14}$

\section{Claims}

Older doctors were generally found to be at 1.1-1.4 times greater risk of malpractice claims or complaints when compared with younger doctors ${ }^{15} 1617$. (One study investigating the predictive impact of age on claims 


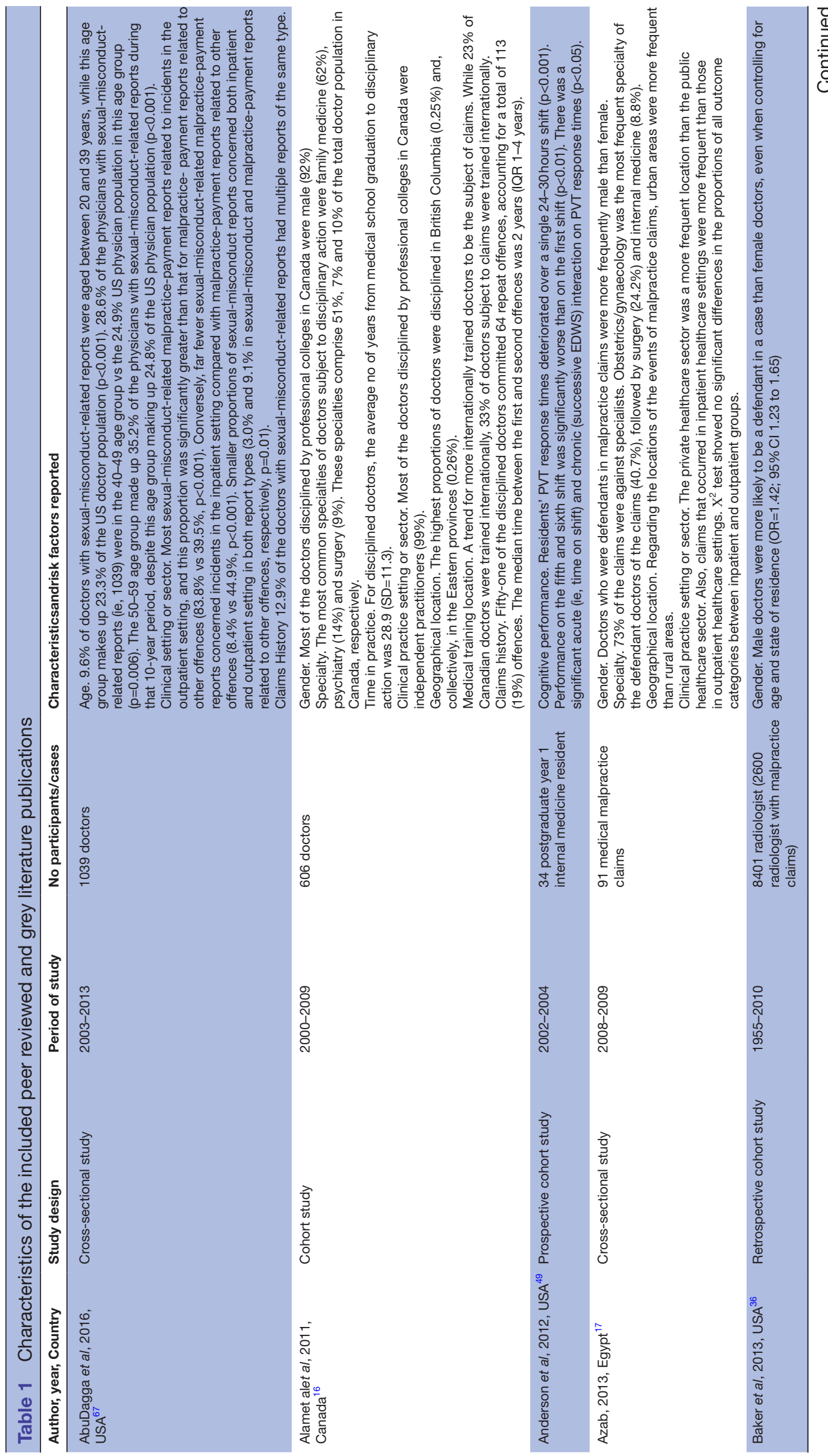




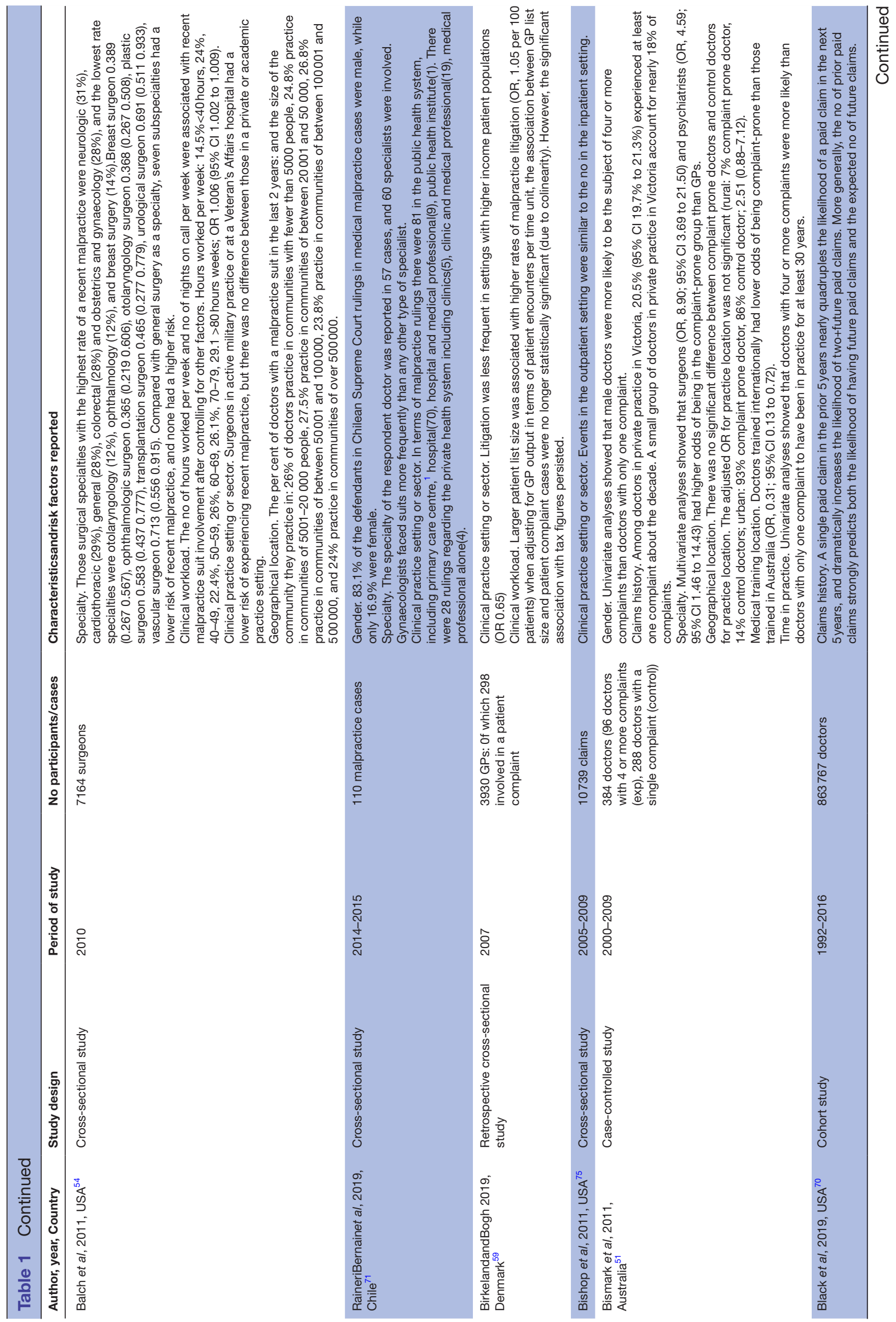




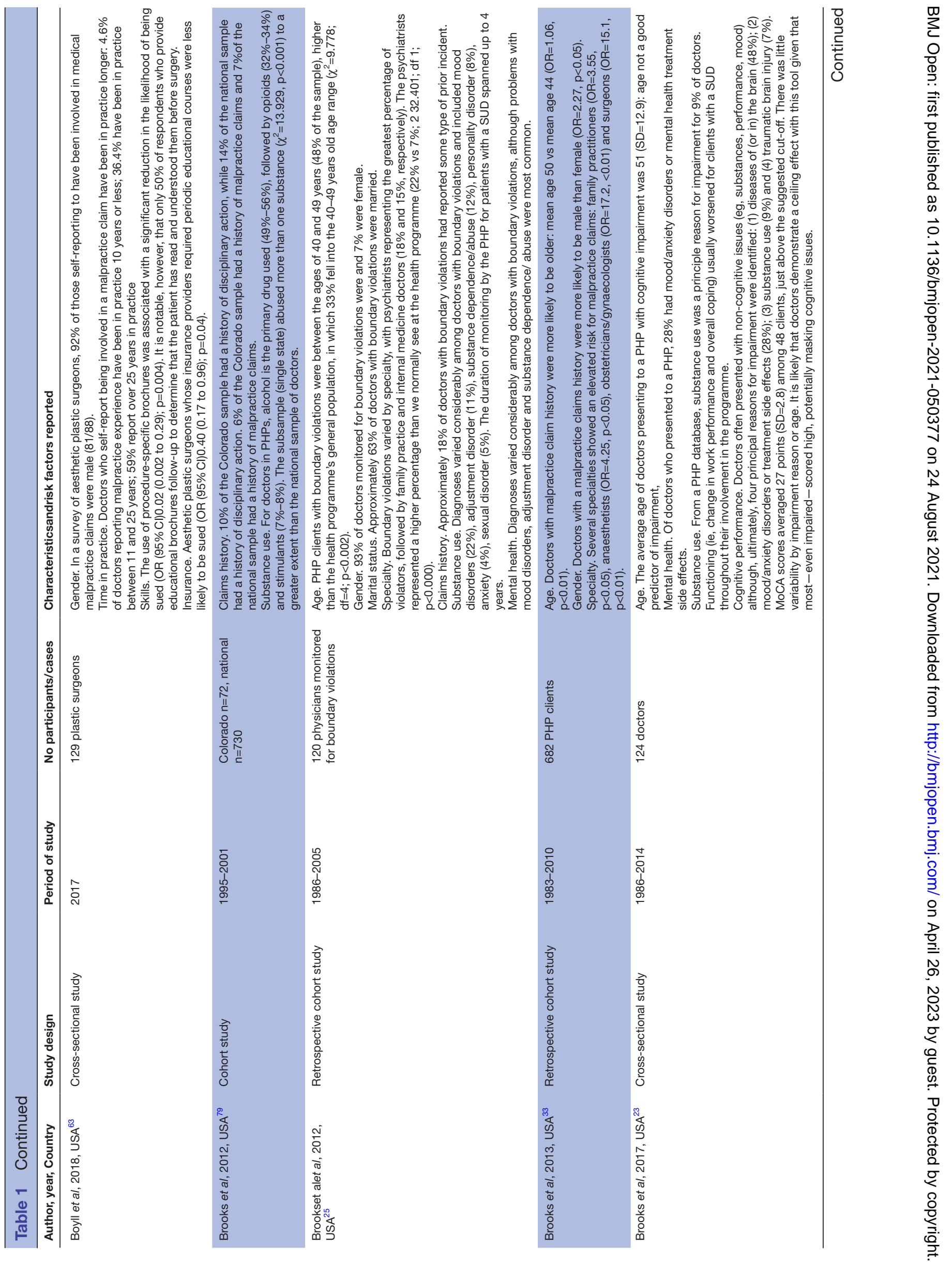




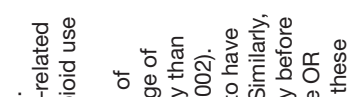

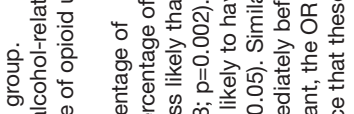

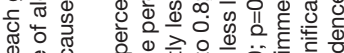

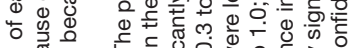

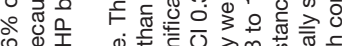

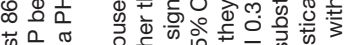

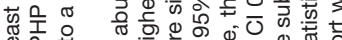

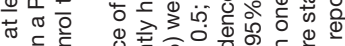

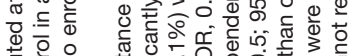

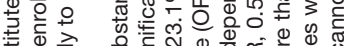

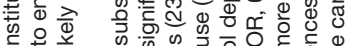

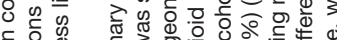
年 कि 的产 w

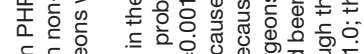

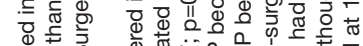

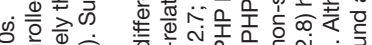

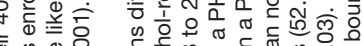

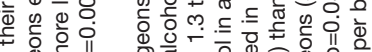

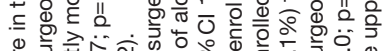

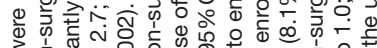

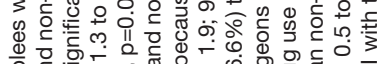

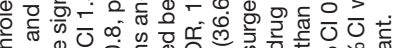

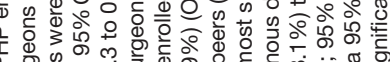

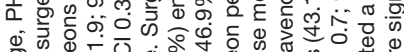

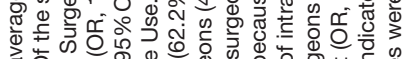

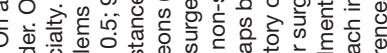

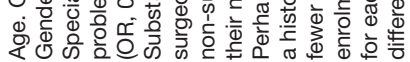

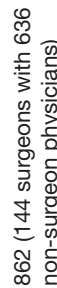

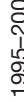



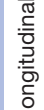

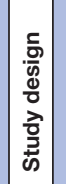

흘

음

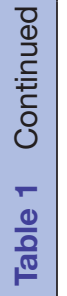

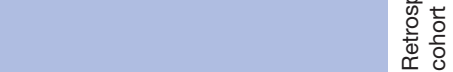

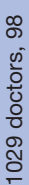

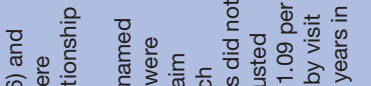

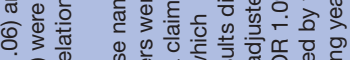

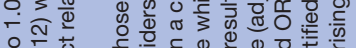

人.

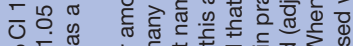

广ั่

ॠ

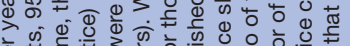

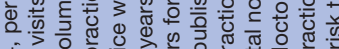

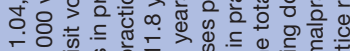

준

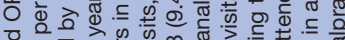

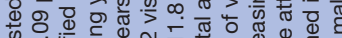

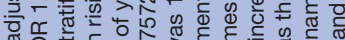

ه응

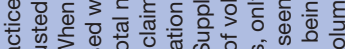

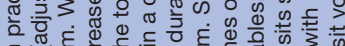

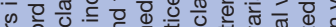

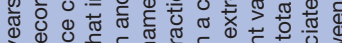

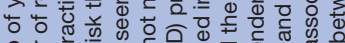

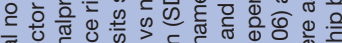

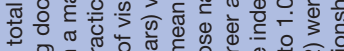

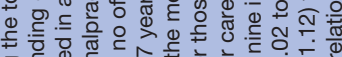

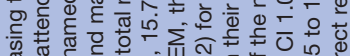

0
D.

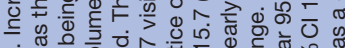

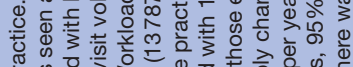

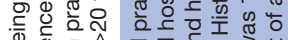

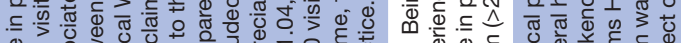

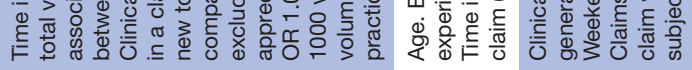

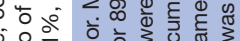

O.

.

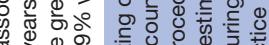

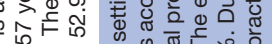




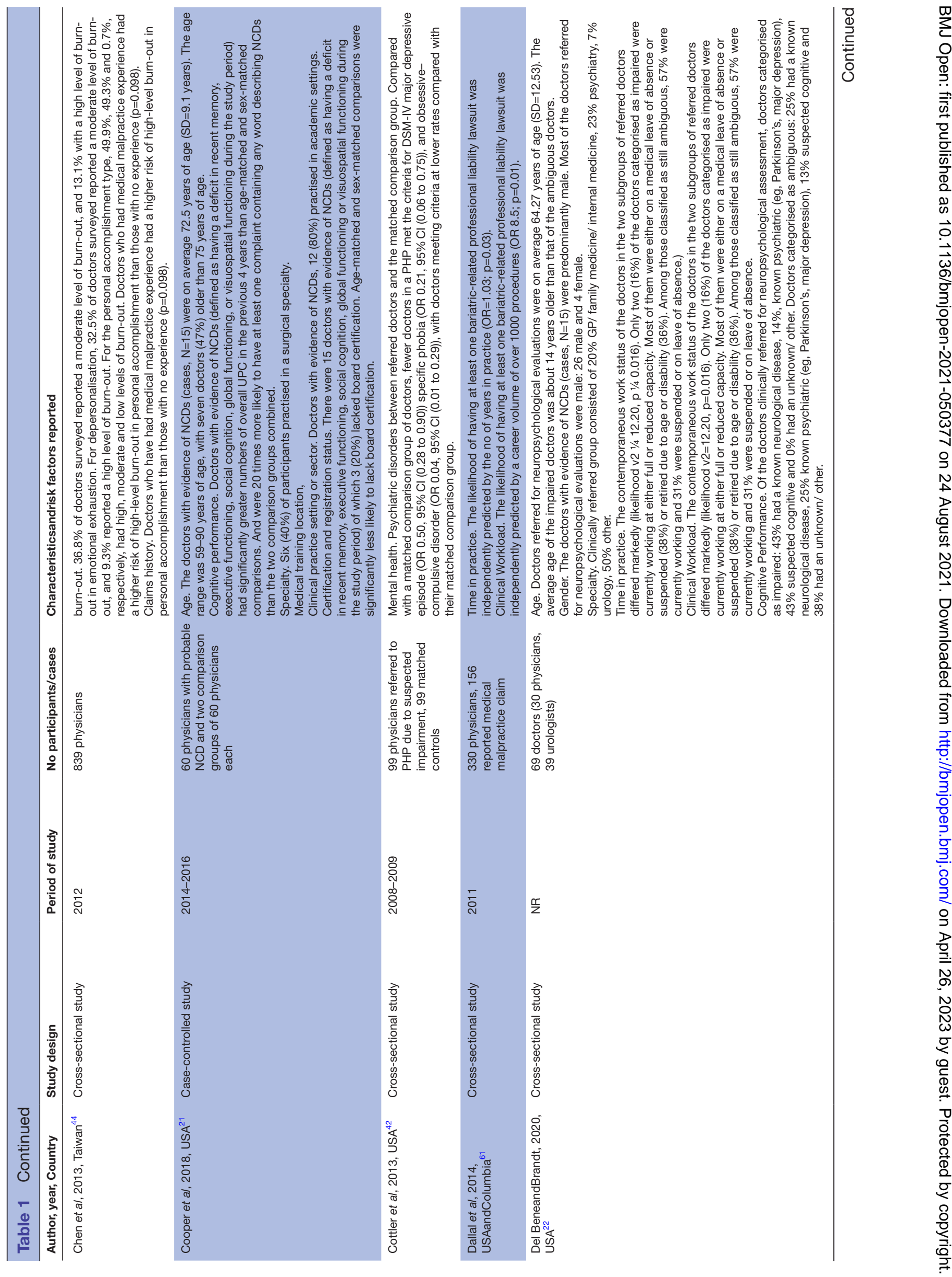




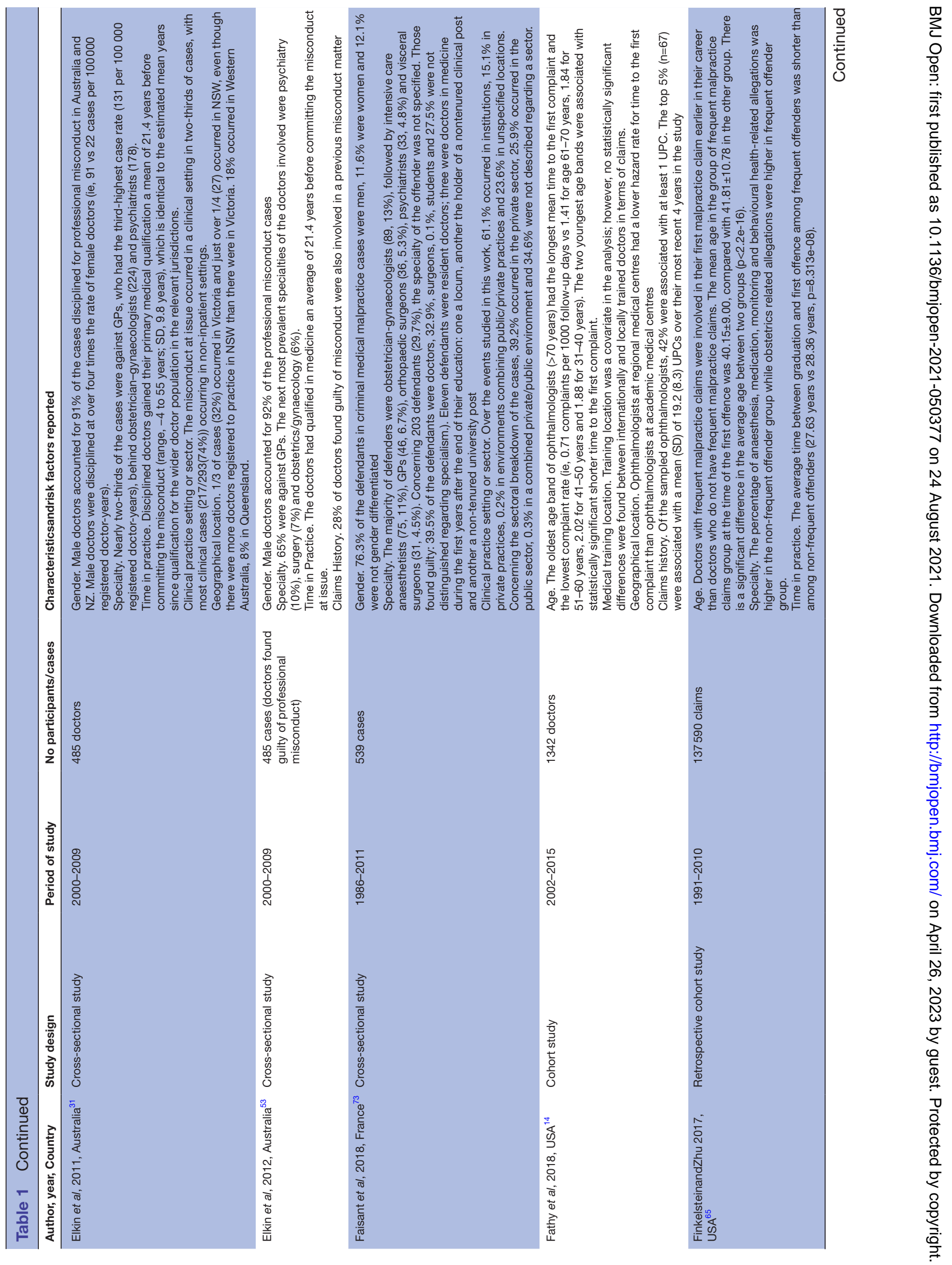




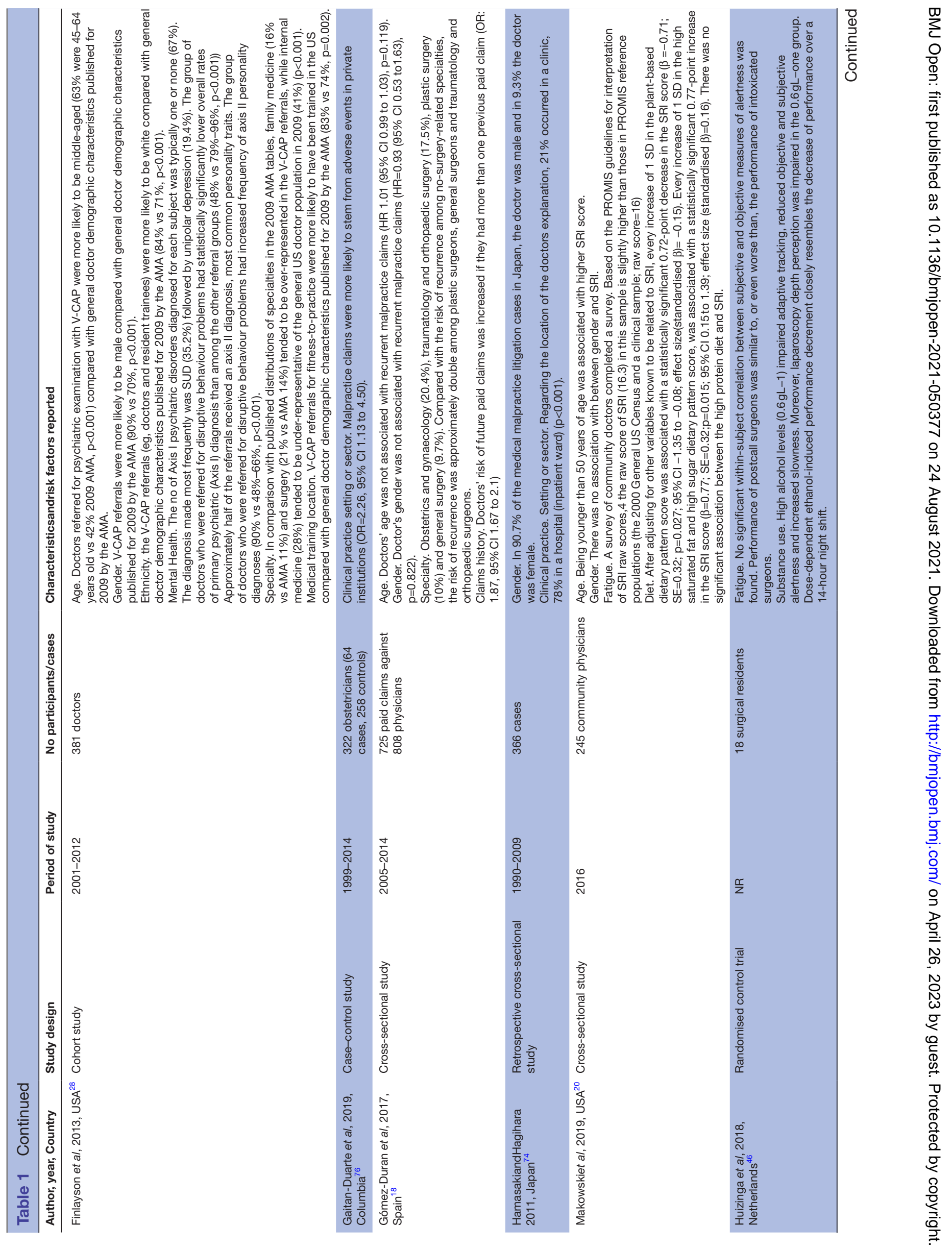




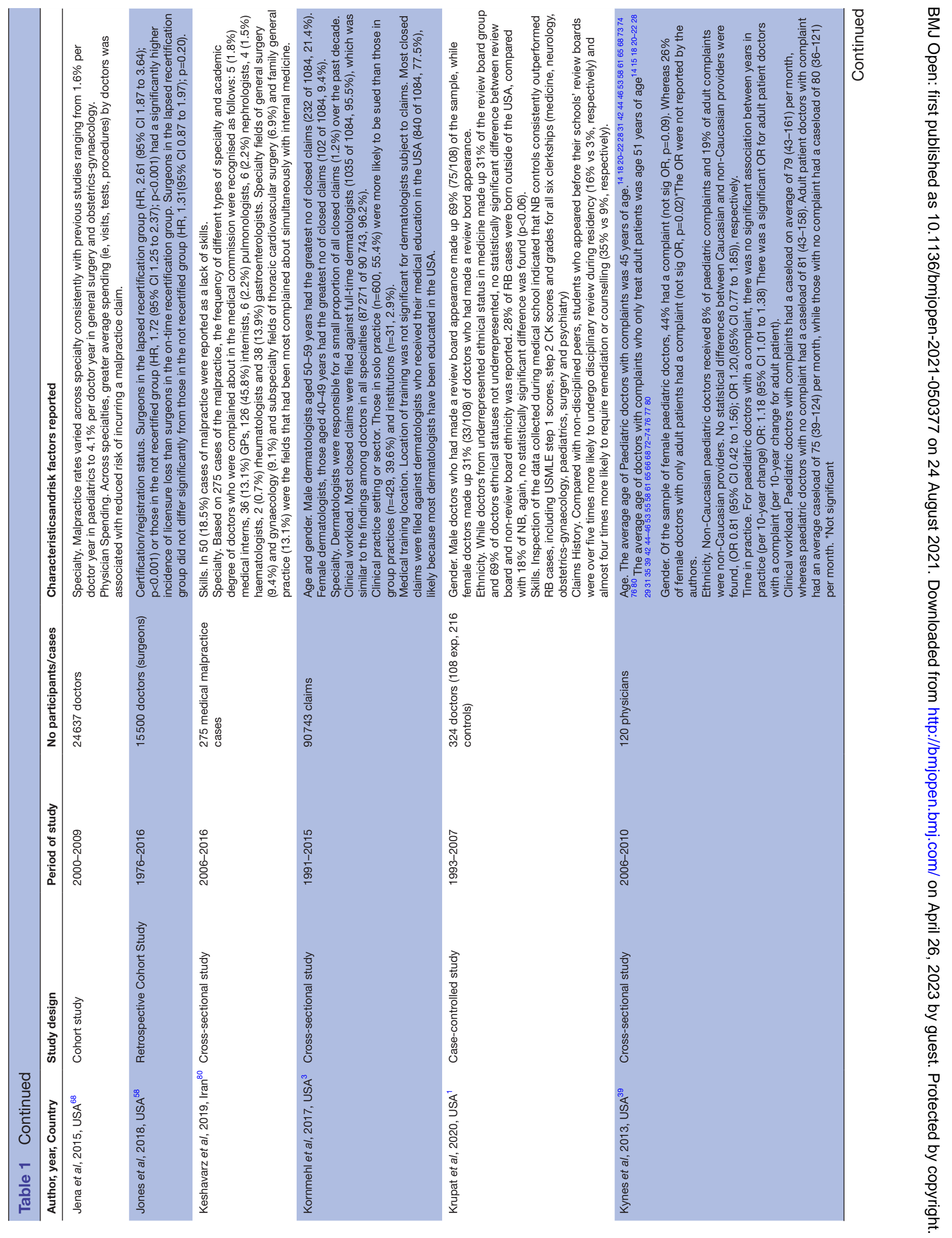




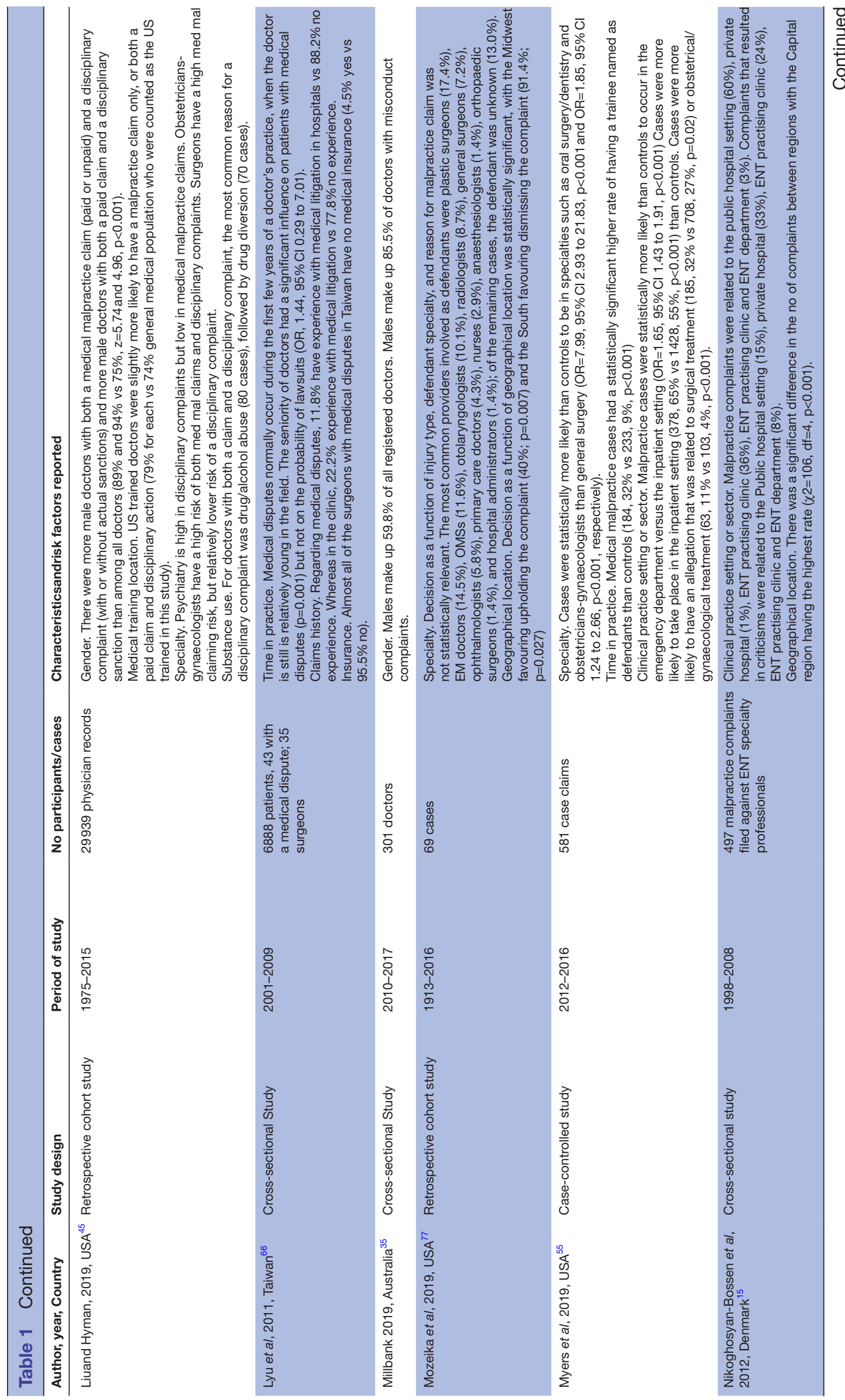




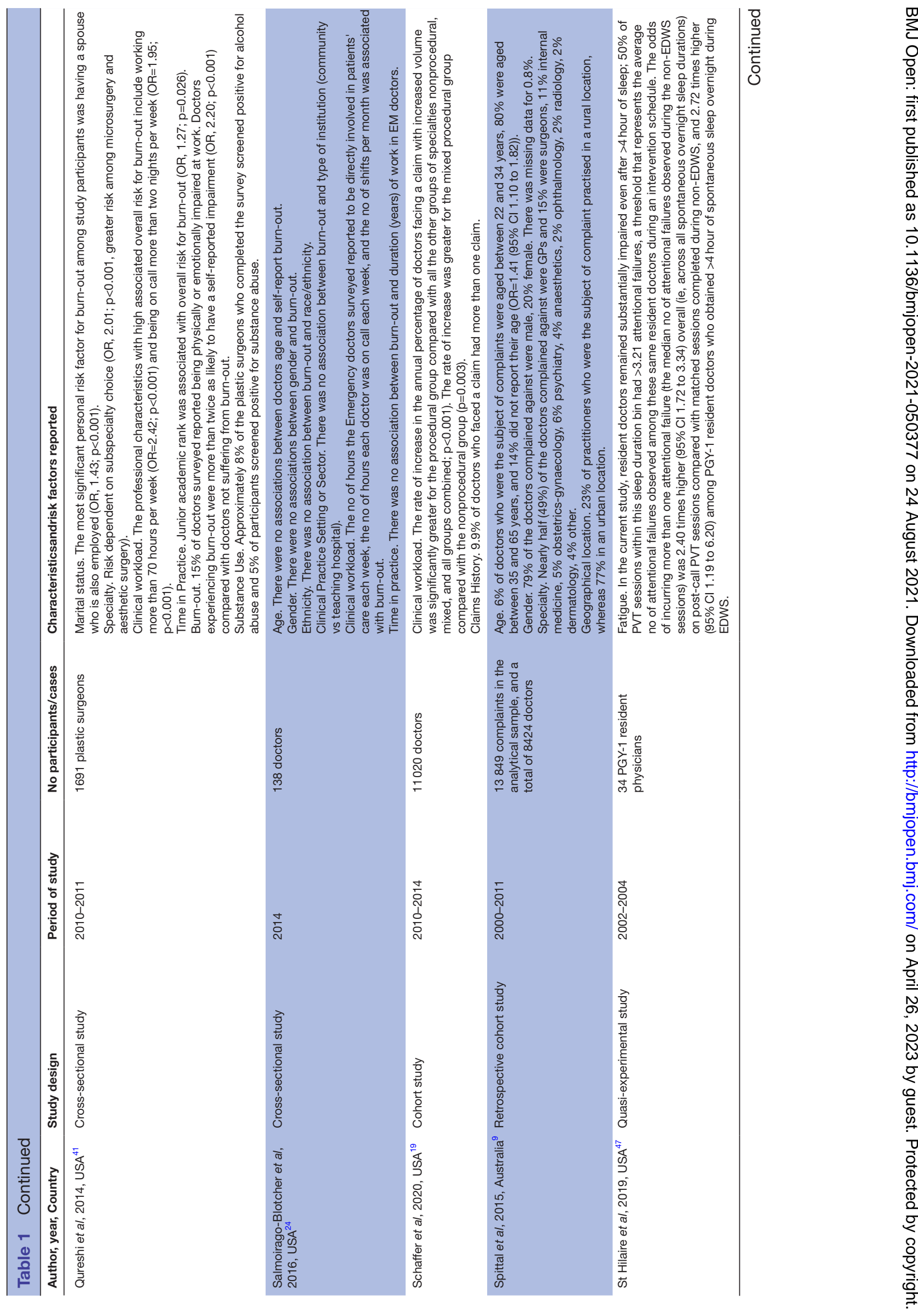




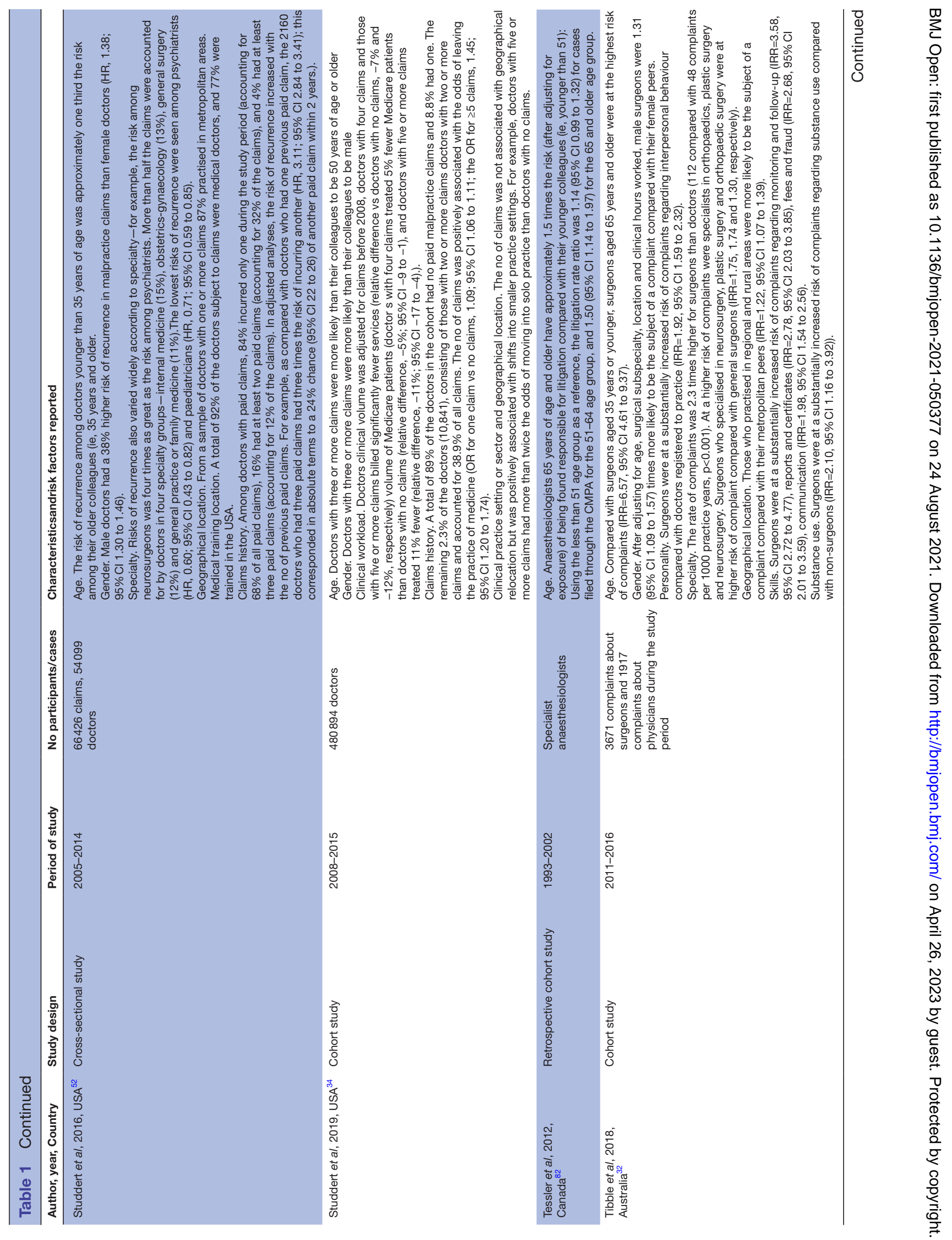




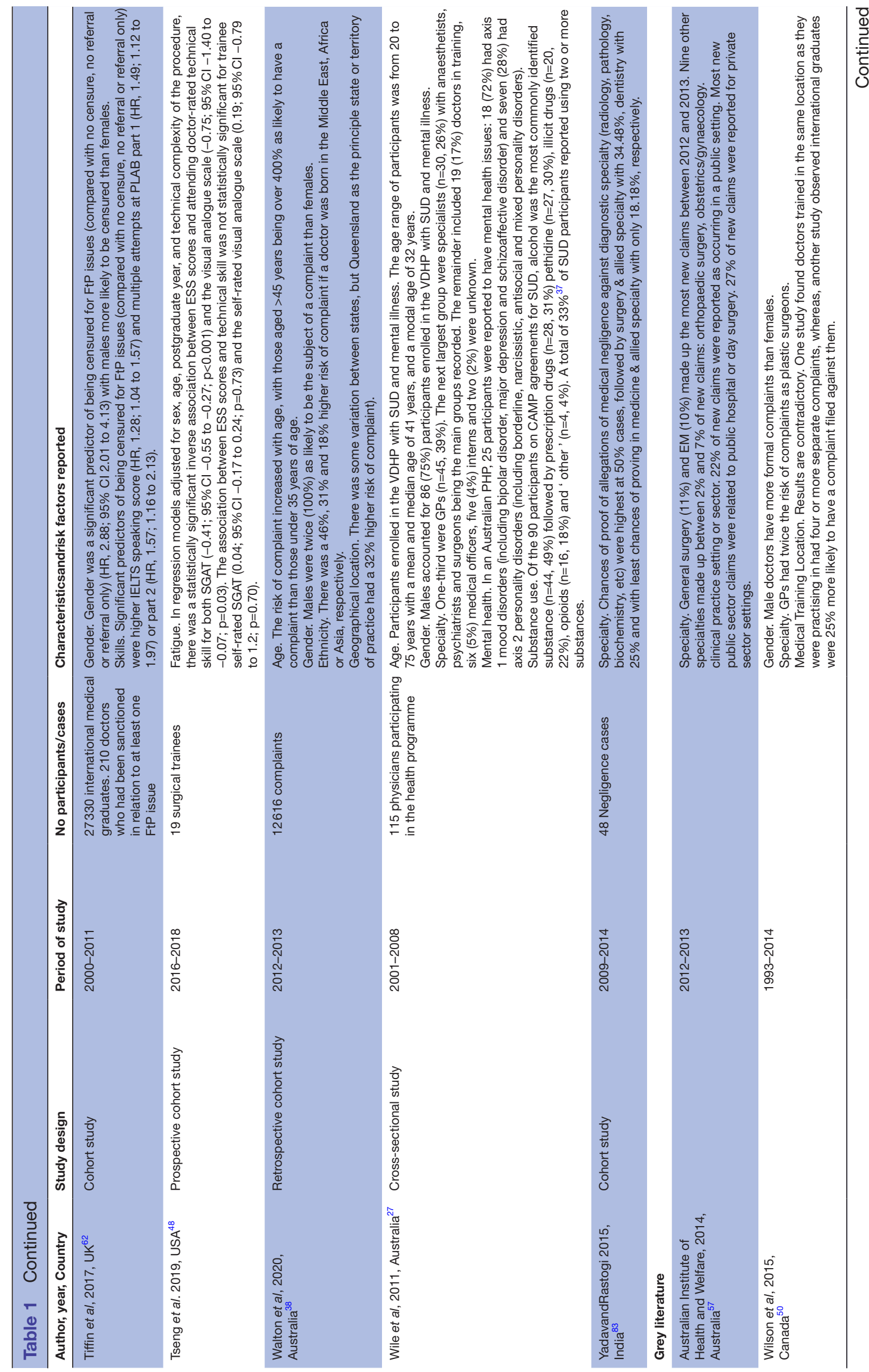



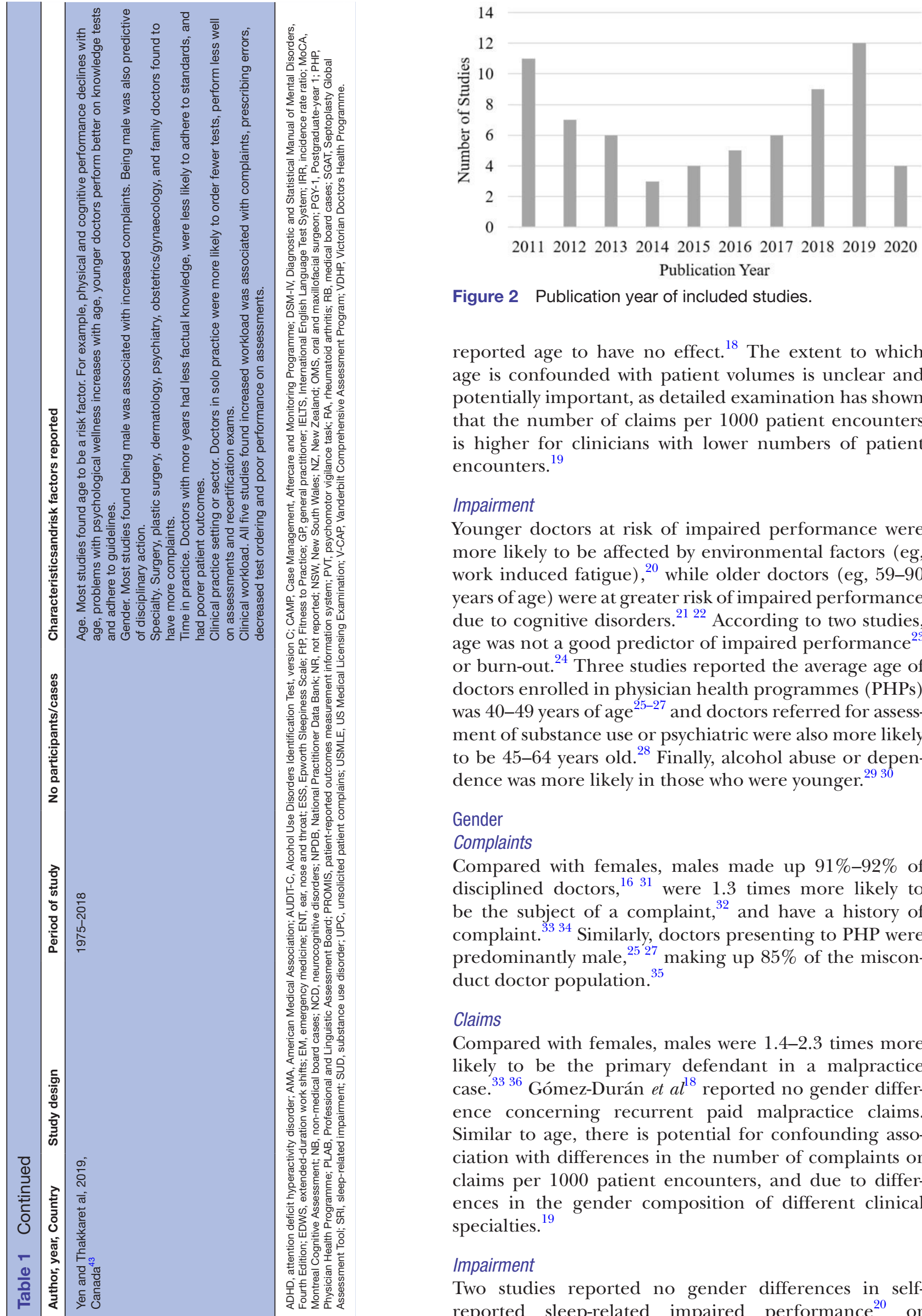

Figure 2 Publication year of included studies.

reported age to have no effect. ${ }^{18}$ The extent to which age is confounded with patient volumes is unclear and potentially important, as detailed examination has shown that the number of claims per 1000 patient encounters is higher for clinicians with lower numbers of patient encounters. ${ }^{19}$

\section{Impairment}

Younger doctors at risk of impaired performance were more likely to be affected by environmental factors (eg, work induced fatigue) ${ }^{20}$ while older doctors (eg, 59-90 years of age) were at greater risk of impaired performance due to cognitive disorders. ${ }^{21}{ }^{22}$ According to two studies, age was not a good predictor of impaired performance ${ }^{23}$ or burn-out. ${ }^{24}$ Three studies reported the average age of doctors enrolled in physician health programmes (PHPs) was 40-49 years of age $\mathrm{e}^{25-27}$ and doctors referred for assessment of substance use or psychiatric were also more likely to be 45-64 years old. ${ }^{28}$ Finally, alcohol abuse or dependence was more likely in those who were younger. ${ }^{29} 30$

\section{Gender}

\section{Complaints}

Compared with females, males made up $91 \%-92 \%$ of disciplined doctors, ${ }^{16}{ }^{31}$ were 1.3 times more likely to be the subject of a complaint, ${ }^{32}$ and have a history of complaint. ${ }^{33} 34$ Similarly, doctors presenting to PHP were predominantly male, ${ }^{25} 27$ making up $85 \%$ of the misconduct doctor population. ${ }^{35}$

\section{Claims}

Compared with females, males were 1.4-2.3 times more likely to be the primary defendant in a malpractice case. ${ }^{336}$ Gómez-Durán et $a l^{18}$ reported no gender difference concerning recurrent paid malpractice claims. Similar to age, there is potential for confounding association with differences in the number of complaints or claims per 1000 patient encounters, and due to differences in the gender composition of different clinical specialties. $^{19}$

\section{Impairment}

Two studies reported no gender differences in selfreported sleep-related impaired performance ${ }^{20}$ or 
Table 2 Results of the critical appraisal

\section{Randomised controlled trials}

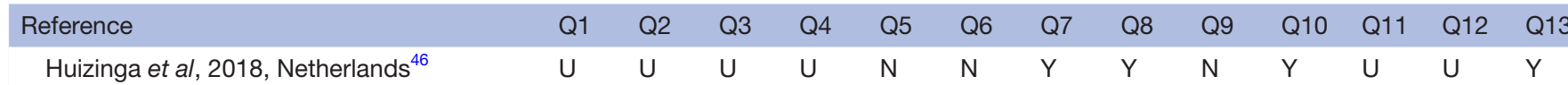

\section{Cohort studies}

Reference

Alam et al, 2011, Canada ${ }^{16}$ Anderson et al, 2012, USA ${ }^{49}$ Baker et al, 2013, USA ${ }^{36}$ Black et al, 2019, USA ${ }^{70}$ Brooks et al, 2013, USA ${ }^{33}$ Brooks et al, 2012, USA ${ }^{25}$ Brooks et al, 2012, USA ${ }^{79}$ Buhl et al, 2011, USA ${ }^{26}$ Fathy et al, 2018, USA ${ }^{14}$ Finkelstein and Zhu 2017, USA ${ }^{65}$ Finlayson et al, 2013, USA ${ }^{28}$ Jena et al, 2015, USA ${ }^{68}$ Jones et al, 2018, USA ${ }^{58}$ Kornmehl et al, 2017, USA ${ }^{3}$ Liu and Hyman 2019, USA ${ }^{45}$ Mozeika et al, 2019, USA ${ }^{77}$ Schaffer et al, 2020, USA ${ }^{19}$ Spittal et al, 2015, Australia $^{9}$ Studdert et al, 2019, USA ${ }^{34}$ Tessler et al, 2012, Canada ${ }^{82}$ Tibble et al, 2018, Australia $^{32}$ Tiffin et al, 2017, UK ${ }^{62}$ Tseng et al, 2019, USA ${ }^{48}$ Walton et al, 2020, Australia ${ }^{38}$ Yadav and Rastogi 2015, India ${ }^{83}$

\section{Case-control studies}

Reference

Bismark et al, 2011, Australia ${ }^{51}$

Cooper et al, 2018, USA ${ }^{21}$

Del Bene and Brandt 2020, USA 22

Gaitan-Duarte et al, 2019, Columbia ${ }^{76}$

Krupat et al, 2020, USA ${ }^{1}$

Myers et al, 2019, USA ${ }^{55}$

\section{Quasi-experimental studies}

Reference St Hilaire et al, 2019, USA ${ }^{47}$

\section{Cross-sectional studies}

\section{Reference}

AbuDagga et al, 2016, USA ${ }^{67}$ Azab 2013, Egypt ${ }^{17}$

Balch et al, 2011, USA ${ }^{54}$

Raineri Bernain et al, 2019, Chile ${ }^{71}$

Birkeland and Bogh 2019, Denmark ${ }^{59}$ $\begin{array}{lllllllllll}\text { Q1 } & \text { Q2 } & \text { Q3 } & \text { Q4 } & \text { Q5 } & \text { Q6 } & \text { Q7 } & \text { Q8 } & \text { Q9 } & \text { Q10 } & \text { Q11 }\end{array}$

$\begin{array}{llllllllllll}Y & Y & Y & N & N & N & Y & Y & Y & N A & Y\end{array}$

$\begin{array}{lllllllllll}Y & Y & Y & U & U & Y & Y & Y & N & N & Y\end{array}$

$\begin{array}{lllllllllllll}Y & Y & U & Y & Y & Y & Y & Y & N A & N A & Y\end{array}$

$\begin{array}{lllllllllll}Y & Y & Y & Y & Y & Y & Y & Y & Y & N A & Y\end{array}$

$\begin{array}{lllllllllll}Y & Y & U & Y & Y & Y & Y & Y & N & N A & Y\end{array}$

$\begin{array}{lllllllllll}Y & Y & Y & N & N A & Y & Y & N & N & N & Y\end{array}$

$\begin{array}{llllllllllll}Y & Y & U & Y & Y & U & U & Y & N & Y & U\end{array}$

$\begin{array}{lllllllllll}Y & U & U & N & N & U & U & Y & Y & Y & Y\end{array}$

$\begin{array}{lllllllllll}Y & Y & Y & Y & Y & Y & Y & Y & Y & N A & Y\end{array}$

$\begin{array}{lllllllllll}Y & Y & U & Y & Y & Y & Y & Y & U & N A & Y\end{array}$

$\begin{array}{lllllllllll}U & U & Y & N & N & N & Y & Y & Y & Y & N A\end{array}$

$\begin{array}{lllllllllll}N A & Y & Y & Y & Y & U & Y & Y & U & U & U\end{array}$

$\begin{array}{llllllllllll}Y & Y & Y & U & U & Y & Y & Y & U & N A & Y\end{array}$

$\begin{array}{llllllllllll}Y & Y & Y & N & N & U & Y & Y & U & N A & Y\end{array}$

$\begin{array}{lllllllllll}Y & Y & Y & N & N & Y & Y & Y & Y & Y & Y\end{array}$

$\begin{array}{lllllllllll}N A & Y & U & Y & Y & Y & Y & Y & Y & N A & U\end{array}$

$\begin{array}{lllllllllll}Y & Y & Y & N & N & U & Y & Y & Y & N A & Y\end{array}$

$\begin{array}{lllllllllll}N A & Y & U & Y & Y & Y & Y & Y & Y & N A & Y\end{array}$

$\begin{array}{llllllllllll}N A & Y & Y & Y & Y & U & Y & Y & Y & N A & Y\end{array}$

$\begin{array}{lllllllllll}Y & Y & Y & Y & Y & Y & Y & N & N & N & Y\end{array}$

$\begin{array}{lllllllllll}Y & Y & Y & Y & Y & U & U & Y & U & N A & Y\end{array}$

$\begin{array}{llllllllllll}Y & Y & Y & Y & Y & Y & Y & N & N & N & Y\end{array}$

$\begin{array}{lllllllllll}N A & Y & U & Y & Y & U & Y & U & U & N A & Y\end{array}$

$\begin{array}{lllllllllll}Y & Y & U & Y & Y & Y & Y & Y & N & N A & Y \\ N A & Y & Y & U & U & U & U & Y & Y & N A & Y\end{array}$

\begin{tabular}{|c|c|c|c|c|c|c|c|c|c|}
\hline Q1 & Q2 & Q3 & Q4 & Q5 & Q6 & Q7 & Q8 & Q9 & Q10 \\
\hline$Y$ & $Y$ & $Y$ & $Y$ & $Y$ & $\mathrm{~N}$ & $\mathrm{~N}$ & $Y$ & $Y$ & $Y$ \\
\hline Y & $Y$ & $Y$ & $Y$ & $Y$ & $Y$ & $Y$ & $Y$ & $Y$ & $Y$ \\
\hline $\mathrm{N}$ & $\mathrm{N}$ & $\mathrm{N}$ & $Y$ & $Y$ & $\mathrm{~N}$ & $\mathrm{~N}$ & $Y$ & $Y$ & $Y$ \\
\hline Y & $Y$ & $Y$ & Y & $Y$ & $Y$ & Y & $Y$ & U & $Y$ \\
\hline Y & $Y$ & $\mathrm{Y}$ & $\mathrm{Y}$ & $\mathrm{Y}$ & $\mathrm{N}$ & $\mathrm{N}$ & $Y$ & $Y$ & $\mathrm{Y}$ \\
\hline Y & $Y$ & $Y$ & $Y$ & $Y$ & $\mathrm{~N}$ & $\mathrm{~N}$ & $Y$ & $Y$ & $Y$ \\
\hline
\end{tabular}

$\begin{array}{lllllllll}\text { Q1 } & \text { Q2 } & \text { Q3 } & \text { Q4 } & \text { Q5 } & \text { Q6 } & \text { Q7 } & \text { Q8 } & \text { Q9 } \\ \mathrm{Y} & \mathrm{Y} & \mathrm{Y} & \mathrm{N} & \mathrm{N} & \mathrm{U} & \mathrm{Y} & \mathrm{U} & \mathrm{Y}\end{array}$

$\begin{array}{llllllll}\text { Q1 } & \text { Q2 } & \text { Q3 } & \text { Q4 } & \text { Q5 } & \text { Q6 } & \text { Q7 } & \text { Q8 }\end{array}$

$\begin{array}{lllllllll}Y & U & Y & U & N & N & Y & Y\end{array}$

$\begin{array}{llllllll}Y & Y & Y & Y & N & N & Y & Y\end{array}$

$\begin{array}{llllllll}Y & Y & U & U & Y & Y & Y & U\end{array}$

$\begin{array}{llllllll}U & U & Y & Y & N & N & U & U \\ Y & Y & U & Y & Y & Y & U & Y\end{array}$


Table 2 Continued

\begin{tabular}{|c|c|c|c|c|c|c|c|c|}
\hline Bishop et al, 2011, USA ${ }^{75}$ & $\mathrm{Y}$ & U & $\mathrm{Y}$ & U & $\mathrm{N}$ & $\mathrm{N}$ & $\mathrm{Y}$ & $\mathrm{Y}$ \\
\hline Boyll et al, 2018, USA ${ }^{63}$ & Y & Y & $\mathrm{N}$ & $\mathrm{N}$ & $\mathrm{Y}$ & $\mathrm{Y}$ & $U$ & $U$ \\
\hline Brooks et al, 2017, USA ${ }^{23}$ & Y & U & $\mathrm{Y}$ & $\mathrm{Y}$ & $\mathrm{N}$ & $\mathrm{N}$ & $\mathrm{Y}$ & $\mathrm{Y}$ \\
\hline Carlson et al, 2018, USA ${ }^{60}$ & $\mathrm{Y}$ & Y & Y & Y & $\mathrm{Y}$ & Y & $\mathrm{Y}$ & $\mathrm{Y}$ \\
\hline Carney et al, 2016, USA ${ }^{64}$ & Y & Y & $\mathrm{N}$ & $\mathrm{N}$ & Y & Y & $\mathrm{N}$ & $\mathrm{Y}$ \\
\hline Cottler et al, 2013, USA ${ }^{42}$ & $\mathrm{Y}$ & $\mathrm{Y}$ & $\mathrm{Y}$ & Y & $\mathrm{N}$ & $\mathrm{N}$ & $\mathrm{Y}$ & $\mathrm{Y}$ \\
\hline Dallal et al, 2014, USA and Columbia ${ }^{61}$ & $\mathrm{Y}$ & U & $\mathrm{N}$ & $\mathrm{N}$ & $\mathrm{Y}$ & $\mathrm{Y}$ & $\mathrm{N}$ & $\mathrm{Y}$ \\
\hline Elkin et al, 2012, Australia ${ }^{53}$ & $\mathrm{Y}$ & $\mathrm{Y}$ & Y & Y & $\mathrm{Y}$ & $\mathrm{Y}$ & $\mathrm{Y}$ & $\mathrm{Y}$ \\
\hline Elkin et al, 2011, Australia ${ }^{31}$ & Y & Y & Y & Y & $\mathrm{Y}$ & $\mathrm{N}$ & $\mathrm{Y}$ & $\mathrm{Y}$ \\
\hline Makowski et al, 2019, USA ${ }^{20}$ & $\mathrm{~N}$ & Y & Y & Y & $\mathrm{Y}$ & Y & Y & $U$ \\
\hline Keshavarz et al, 2019, Iran ${ }^{80}$ & $\mathrm{Y}$ & Y & $\mathrm{Y}$ & $\mathrm{Y}$ & $\mathrm{N}$ & $\mathrm{N}$ & $\mathrm{Y}$ & $\mathrm{Y}$ \\
\hline Kynes et al, 2013, USA ${ }^{39}$ & Y & Y & $U$ & $\mathrm{~N}$ & $\mathrm{~N}$ & $\mathrm{~N}$ & $\mathrm{Y}$ & $\mathrm{Y}$ \\
\hline Lyu et al, 2011, Taiwan ${ }^{66}$ & $\mathrm{Y}$ & Y & $U$ & $\mathrm{Y}$ & $U$ & $\mathrm{~N}$ & $u$ & $\mathrm{Y}$ \\
\hline Millbank 2019, Australia ${ }^{35}$ & $\mathrm{Y}$ & Y & Y & Y & $\mathrm{N}$ & $\mathrm{N}$ & $\mathrm{Y}$ & $\mathrm{Y}$ \\
\hline Nikoghosyan-Bossen et al, 2012, Denmark ${ }^{15}$ & $\mathrm{Y}$ & $\mathrm{U}$ & U & $\mathrm{Y}$ & $\mathrm{N}$ & $\mathrm{N}$ & $\mathrm{Y}$ & $\mathrm{Y}$ \\
\hline Oreskovich et al, 2012, USA ${ }^{30}$ & Y & Y & Y & Y & $\mathrm{N}$ & $\mathrm{N}$ & $\mathrm{Y}$ & $\mathrm{Y}$ \\
\hline Oreskovich et al, 2015, USA ${ }^{29}$ & Y & Y & $\mathrm{N}$ & Y & $\mathrm{Y}$ & $\mathrm{Y}$ & $\mathrm{Y}$ & Y \\
\hline Oshel and Levitt 2016, USA ${ }^{81}$ & U & U & Y & Y & $\mathrm{Y}$ & $\mathrm{N}$ & $\mathrm{Y}$ & $\mathrm{Y}$ \\
\hline
\end{tabular}

N, no; NA, not available; U, unclear; Y, yes.

burn-out. ${ }^{24}$ While males represented over half $(57 \%-$ $86 \%$ ) the doctors presenting to PHPs, ${ }^{26}{ }^{37}$ one study reported female surgeons had almost twice the rates of alcohol abuse or dependence as male surgeons. ${ }^{30}$ Doctors with evidence of neurocognitive disorders or referred for psychiatric examination were predominantly male $(90 \%$ vs $70 \%) .{ }^{21} 2228$

\section{Ethnicity}

\section{Complaints}

One Australian study reported an 18\%, 31\% and $46 \%$ higher risk of complaint if a doctor was born in Asia, Africa or the Middle East, respectively. ${ }^{38}$ In contrast, two studies from the USA reported no statistical differences between Caucasian and non-Caucasian doctors. ${ }^{139}$

\section{Impairment}

One study reported referrals for assessment of Caucasian doctors were proportionately higher $(84 \%$ vs $71 \%)$ when compared with general doctor characteristics group published by the American Medical Association. ${ }^{28}{ }^{40}$ Whereas a second study did not find an association between ethnicity and burn-out, as a predictor of impaired performance. ${ }^{24}$

Marital status

\section{Claims}

No association was found between doctor malpractice and marital status. ${ }^{25} 33$

\section{Impairment}

Partnered doctors were 1.3-2 times more likely to suffer alcohol abuse or dependence, and doctors whose spouse was also employed were 1.43 times more likely to suffer burn-out-related impaired performance. ${ }^{29} 3041$

Mental health, personality and burn-out

Complaints

One study reported that surgeons were 1.9 times more likely than physicians to have complaints attributed to 


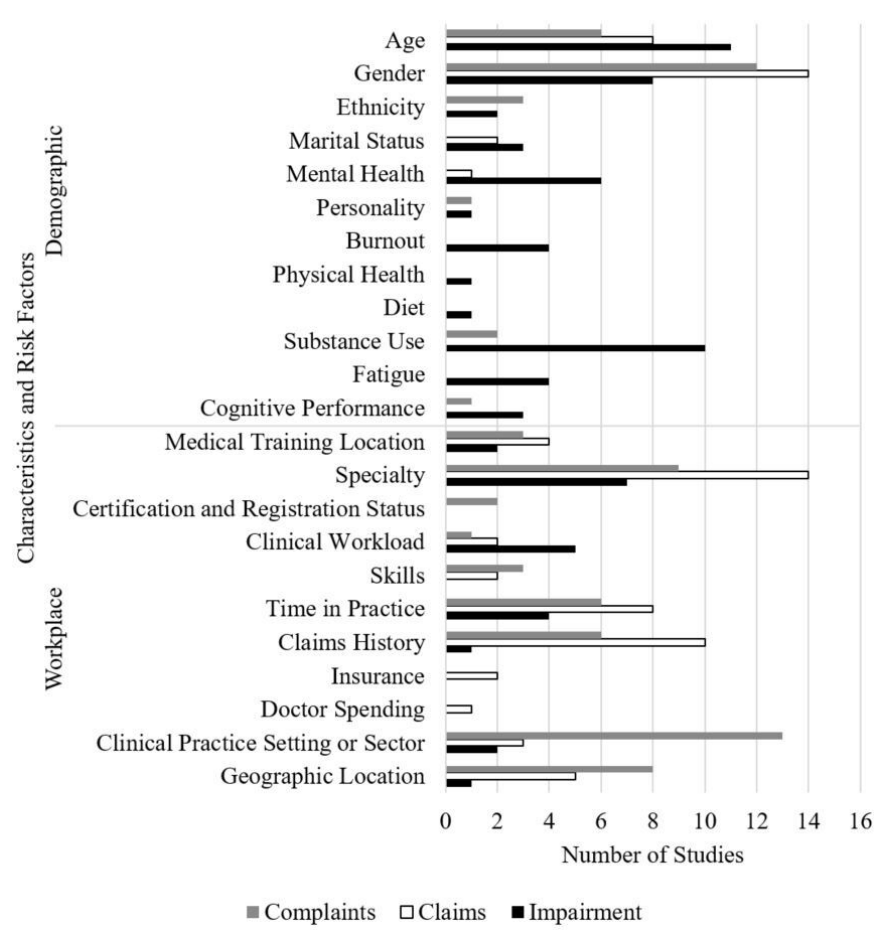

Figure 3 The number of publications for each factor associated with doctors at risk of malpractice claims and/or complaints, or impaired performance.

interpersonal behaviour problems (eg, complaints about bullying, discrimination). ${ }^{32}$

\section{Claims}

One study reported that doctors with malpractice claims had a variety of mental health diagnoses with the most common being mood disorders $(12 \%)$ and substance abuse $(12 \%) .{ }^{25}$

\section{Impairment}

A small number of studies addressed mental health, ${ }^{23} 2728374243$ personality ${ }^{23}$ and burn-out. ${ }^{304144} \mathrm{~A}$ higher proportion of doctors with impaired performance had mental health issues including bipolar disorder, depression and anxiety ${ }^{2327} 37$ and problems with mental health increased with age. ${ }^{43}$ Doctors and their peers were often aware of the presence of an underlying issue with one-third of doctors self-referring for cognitive assessment. ${ }^{23}$ Higher levels of burn-out (eg, depersonalisation) related impaired performance was reported by three of four studies ${ }^{304144}$ and associated with more hours of direct patient care, more hours on-call and more shifts per month. ${ }^{24}$ Doctors with emotional exhaustion also showed greater alcohol use or dependence. ${ }^{30}$

\section{Physical health and diet}

\section{Impairment}

While physical health declined with age ${ }^{43}$ one study found that, for doctors self-referring to a PHP, mental health problems were more common than physical (26\% vs $1 \%) .{ }^{37}$ Doctors with diets that were high in plant-based foods and low in saturated fat and added sugars had less sleep-related impaired performance. ${ }^{20}$

\section{Substance use}

Complaints

In Australia, compared with physicians, surgeons were at 2.1 times higher risk of complaints regarding substance use. ${ }^{32}$ In Indiana, USA, drug or alcohol abuse and drug diversion were the most common reasons for a disciplinary complaint. ${ }^{45}$

\section{Claims}

One study found no relationship between either malpractice or maladaptive behaviours for a sample of emergency medicine doctors, reporting that doctors who attended worship services had an odds of 0.4 of maladaptive behaviours including smoking (10\%), marijuana or cocaine use $(10 \%)$ and alcohol use. ${ }^{24}$

\section{Impairment}

Data from PHPs in multiple jurisdictions found alcohol was the most commonly used substance, with reports between $8 \%$ and $15 \%$ of medical practitioners screened positive for alcohol abuse. ${ }^{37}$ The secondary consequences of alcohol use were reported to include addiction problems, ${ }^{3746}$ mental and physical impaired performance (eg, reduced alertness, impaired adaptive tracking) ${ }^{3046}$ and recent major medical errors. ${ }^{30}$

\section{Fatigue}

\section{Impairment}

In general, community doctors have a slightly higher sleep-related impairment score compared with reference populations (ie, the 2000 General US Census). ${ }^{20}$ Surgeons' performance post-on-call shift (ie, when a clinician is able to be contacted to provide care if necessary, but not formally on duty) was similar to, or worse than, the performance of intoxicated surgeons to a degree where dose-dependent ethanol-induced performance closely resembled the decrease of performance over a 14-hour night shift. ${ }^{46}$ Similarly, a study from the $\mathrm{USA}^{47}$ reported that doctors remained impaired (eg, attentional failures) even after over 4 hours of sleep. This study also reported an inverse relationship between fatigue and technical skill. ${ }^{48}$

\section{Cognitive performance}

\section{Complaints}

One study found doctors with neurocognitive disorders (defined as having a deficit in recent memory, executive functioning, social cognition, global functioning or visuospatial functioning during the study period) had more unsolicited patient complaints compared with agematched and sex-matched comparators. ${ }^{21}$

\section{Impairment}

Cognitive performance is affected by age $e^{43}$ and deteriorates throughout the course of successive shifts, being significantly worse in the fifth and sixth shift, and/or 
longer shift length. ${ }^{49}$ Doctors presented to PHPs with four principal reasons for impaired performance: (1) brain disease $(48 \%)$; (2) mood/anxiety disorders or treatment side effects (28\%); (3) substance use (9\%) and (4) traumatic brain injury $(7 \%) \cdot{ }^{23}$ Of the doctors clinically referred for neuropsychological assessment, impaired doctors were referred predominantly with known neurological disease, known psychiatric (eg, Parkinson's disease, major depression) or suspected cognitive diagnoses. ${ }^{22}$

\section{Workplace characteristics \\ Medical training location \\ Complaints}

Evidence was mixed on whether locally trained doctors or internationally trained doctors were more subject to complaints. ${ }^{50}$ A Canadian study found that $33 \%$ of doctors subject to claims were trained internationally, while these doctors make up $23 \%$ of the doctor population. ${ }^{16}$ An Australian study found doctors trained internationally had lower odds of being complaint-prone than those trained in Australia. ${ }^{51}$

\section{Claims}

One study found that slightly fewer internationally trained doctors were subject to claims, ${ }^{45}$ and two studies found no difference. $^{1452}$

\section{Impairment}

Through the complaints process, one study identified a greater percentage of internationally trained doctors with a neurocognitive impairment (33\% vs $23 \%),{ }^{21}$ while another study found that relatively fewer internationally trained doctors ( $74 \%$ vs $83 \%$ ) were referred for fitness-topractice concerns. ${ }^{28}$

\section{Specialty}

\section{Complaints}

General practitioners (GPs) and family medicine specialists comprise a large proportion of total complaints (49\%-65\%). ${ }^{9} 165053$ Other high-risk specialties include psychiatry, surgery, obstetrics and gynaecology. ${ }^{16} 315153$ For example, surgeons were noted to be 2.3 times as likely to be subject to complaint compared with GPs. ${ }^{32}$

\section{Claims}

Overall, general surgery had higher risk of malpractice when compared with other surgical subspecialties, ${ }^{18} 54$ however, agreement was not universal. ${ }^{55}$ Grey literature and published academic papers reported GPs and family medicine specialists comprise a large proportion of total claims $(34 \%-62 \%)$ and were up to three times as likely to be subject to claims. ${ }^{33} 505556$ Other high-risk specialties include psychiatry, obstetrics and gynaecology, which were noted to be between four and 17 times as likely to be subject to malpractice claims compared with GPs. ${ }^{33} 57$

\section{Impairment}

According to the location of the study, specialty as a risk factor varied between countries. In Australia, impaired performance was less of a risk for specialists, ${ }^{27}$ while in the USA two studies found a higher relative risk for surgeons, psychiatrists and family medicine doctors. ${ }^{28}{ }^{41} \mathrm{~A}$ further study conducted in the USA found that surgeons were 1.9 times more likely than non-surgeons to enrol in a PHP because of alcohol-related problems ${ }^{26}$ and 0.5 times less likely because of opioid use.

\section{Certification status and clinical workload}

\section{Complaints}

Increased workload was associated with increased complaints, medical errors and poor assessment performance. ${ }^{4350}$ One study did not find a relationship between complaints and clinical load. ${ }^{39}$

\section{Claims}

Licensure loss was associated with lapsed or missed certification. ${ }^{58}$ Greater number of hours worked per week, ${ }^{354}$ greater number of patients seen ${ }^{19} 59-61$ and more years in practice $^{60}$ were associated with a 1.0-1.1 times higher risk of malpractice claims.

\section{Impairment}

Doctors with evidence of neurocognitive disorders were more likely than age-matched and sex-matched doctors to lack board recertification. ${ }^{21}$ The highest percentage of doctors with impaired performance was found in the GP group (39\%), with specialists comprising $26 \% .{ }^{27}$ Doctors working longer hours and additional nights on call had lower prevalence of alcohol abuse, ${ }^{29}{ }^{30}$ but 2.0-2.4 times higher risk of burn-out. ${ }^{241}$

\section{Skills}

\section{Complaints}

Poor performance on medical knowledge and licensing exams $^{1}$ and language assessments ${ }^{62}$ were predictors of complaints and fitness to practice issues. There was a 3.6 times greater risk of complaints during patient monitoring and follow-up, ${ }^{32}$ however, a 2019 Canadian report found that the majority of complaints were related to the clinical aspects of care, rather than issues with communication and/or unethical or improper behaviour. ${ }^{43}$

\section{Claims}

The use of procedure-specific patient education brochures reduced the likelihood of surgeons being sued. ${ }^{63}$

\section{Time in practice}

\section{Complaints}

Frequency of disciplinary cases increased with years in practice $^{4350}$; it has been postulated that those with more years in practice had less factual knowledge and were less likely to adhere to standards. ${ }^{43}$

\section{Claims}

Frequency of malpractice claims increased with years in practice. $^{39} 51606164$ In contrast, three studies reported practitioners with fewer years in practice had higher odds of a malpractice claim or medical litigation..$^{55666}$ 


\section{Impairment}

Junior doctors who spent less time in practice than their senior counterparts were 1.3 times more likely to suffer impaired performance. ${ }^{41}$ However, there was no association between duration of practice and impaired performance. ${ }^{24}$ Sixteen per cent of doctors clinically referred for neuropsychological assessment and subsequently categorised as impaired were currently working full time or at reduced capacity. ${ }^{22}$

\section{Claims history, insurance and doctor spending Complaints}

Between $14 \%$ and $28 \%$ of doctors who receive complaints have experienced complaints previously. ${ }^{165367}$

\section{Claims}

Doctors who were a recipient of a previous claim were reported to be at 1.9 times greater risk of a repeated claim, ${ }^{18}$ even when controlling for age, gender and specialty. ${ }^{18} 52$ US doctors whose insurance providers required participation in educational courses were less likely to be sued. ${ }^{63}$ Greater average doctor spending (eg, visits, tests, procedures) was associated with reduced risk of malpractice claim. ${ }^{68} 69$ The likelihood of multiple claims increased with the duration of study coverage, the calendar year and the country. 118345270 The potential confounding effect of doctor activity level was not accounted for in the aforementioned studies.

\section{Impairment}

Previous malpractice experience was associated with greater risk of impaired performance. ${ }^{44}$

\section{Clinical practice setting or sector Complaints}

One study reported complaints were more frequent for inpatient settings, ${ }^{15}$ while two studies reported that complaints were more frequent for doctors working in outpatient settings and independent practice. ${ }^{31} 67$ Doctors in solo/independent practice were more likely to be disciplined than those in group practices. ${ }^{16}$ One study reported a higher percentage of complaints against the private sector compared with the public system. ${ }^{15}$

\section{Claims}

Seven studies reported claims were more frequent for inpatient settings, ${ }^{15} 1755$ 71-74 while two studies reported that claims were more frequent for doctors working in outpatient settings and independent practice. ${ }^{3} 54$ One study reported a similar number of claims regarding inpatient and outpatient settings. ${ }^{75}$ Doctors in solo/independent practice were more likely to be sued or have recent claims than those in group practices, ${ }^{3} 54$ they were also more likely to do less well on assessments and recertification exams. ${ }^{43}$ Conversely, those with five or more claims were more likely to move into solo practice than doctors with no claims. $^{34}$

Three studies reported a higher percentage of ${ }^{15}$ claims $^{73}$ against the private sector compared with the public system, with only one study reporting the opposite. $^{71}$

\section{Impairment}

No relationship was found between burn-out and the type of institution the doctor practised in. ${ }^{24}$ Of doctors with evidence of neurocognitive disorders, $80 \%$ practised in academic settings. ${ }^{21}$

\section{Geographical location}

\section{Complaints}

While three studies reported that doctors practising in metropolitan areas were at greater risk of complaint than doctors practising in regional and remote areas, ${ }^{9} 1532$ one study reported no relationship between geographical location and frequency of claims. ${ }^{51}$ Conversely, doctors (ophthalmologists) in regional medical centres had a longer time to the first complaint than those practising at academic medical centres. ${ }^{14}$ In the USA, complaints were more likely to be upheld in the Midwest than in the South. ${ }^{77}$ In Australia, doctors practising in Queensland or Victoria had a higher risk of a complaint than in other states. $^{3138}$

\section{Claims}

While two studies reported that doctors practising in metropolitan areas were at greater risk of malpractice claim than doctors practising in regional and remote areas, ${ }^{17} 52$ two studies reported no relationship between geographical location and frequency of claims. ${ }^{3454}$

\section{Impairment}

No relationship was found between the size of a doctor's practice community and the rate of alcohol abuse/ dependence. $^{29}$

\section{DISCUSSION}

We examined the research evidence provided in peerreviewed and grey literature identifying and characterising high-risk practitioners. This systematic review found older age, males, longer time in practice, having a history of claims, specialty and heavier clinical workload to be most frequently cited as risk factors for malpractice claims or complaints. The issue of risk factors and predicting complaints and claims is far more complex and context specific than a list of factors suggests. For example, in some countries, it may not be possible to pursue lawsuits for compensation, rather compensation for poor outcomes maybe settled through non-legal means. Differences in settlement practices may explain differences in the rate of complaints and claims between countries. The evidence examining risk factors for impaired performance was limited and likely subject to bias due to analysis primarily based on self-reported measures. ${ }^{24} 304144$ For example, studies that used objective measures to record burn-out found fatigue and substance use to impair clinical practice, ${ }^{30} 46$ whereas studies using self-reported measures of burn-out found impaired performance stemming from 
burn-out manifested in exhaustion, depersonalisation of patients, and reduced sense of personal accomplishment. 24304144

Factors associated with higher risk of malpractice claims or complaint are multifactorial and likely interdependent. For example, one contributing factor to a doctor experiencing burn-out may be higher clinical workload. ${ }^{41}$ High patient load or workload was associated with complaints, medical errors and poor performance on assessment. ${ }^{43} 50$ High workload and fatigue go hand in hand, with fatigue consistently linked to impaired performance (eg, diminished technical skills). ${ }^{20} \quad 46-48$ For example, cognitive performance was found to deteriorate throughout a work shift, and cumulatively worsen over the course of successive shifts. ${ }^{49}$ Impaired performance such as attentional failures can remain even after a period of rest. ${ }^{47}$ Crucially, the decrease in performance/skill due to fatigue is similar to, or greater than performance impairments seen with alcohol intoxication. ${ }^{46}$ While the included studies did not directly link impaired performance with malpractice claims or complaint, it is likely that a diminishment or loss of ability plays a role in a doctor's risk for malpractice claim or complaint.

Medical governance and regulatory bodies (eg, Medical Board of Australia, Federation of State Medical Boards-America) typically collect standard information on doctors (eg, age, gender, specialty) ${ }^{78}$ It is not practical or ethical for governance or regulatory bodies to collect and apply all 23 factors identified by the review. Instead, factors that are not currently collected by medical governance and regulatory bodies could perhaps be collected by employers, medical defence insurers and doctor health programmes which target high risk members. The need to collect information needs to be balanced and justified against registrants' right to privacy. While no single factor was a strong predictor of risk of claims, complaint or impaired performance, collection of data on all factors would establish the extent to which each factor contributes to a doctor's risk within the local context.

The findings highlight the need to include medical practitioner voices in the discourse on risk and prevention and the contribution of a context-informed perspective that includes awareness of demographic, workplace, social and legal effects. New ways of supporting doctors might be developed, using risk factor data to reduce adverse events and patient harm. For example, widespread education, peer support, mentoring and early intervention for factors associated with complaints, malpractice and impaired performance may facilitate early identification of at-risk doctors, including identification by colleagues, supervisors and patients. It may also help doctors recognise the factors in their own practice that elevates their risk of complaints, malpractice or impaired performance and encourage behavioural change. For instance, understanding that high patient load is a risk factor, medical practitioners might make strategic decisions around the volume of work they choose to take on. Similarly, employers might use the understanding that high patient volume puts medical practitioners and patients at risk to establish safe and effective workload policies and organisational culture practices. To provide a clearer picture on a doctor's risk, baseline data for the normal population should also be reported alongside doctor population data. Reporting data from both populations would enable absolute rather than relative risk to be determined and facilitate pooling of data for analysis.

\section{Strengths and limitations}

A comprehensive search and rigorous review processes was used to search and appraise studies from a range of academic databases and grey literature sources. We were unable to pool data for analyses due to the heterogeneity of data collection and analysis methods. The majority of studies investigating risk of malpractice claim or complaint were descriptive and involved no comparator groups, limiting the ability to generalise study findings to the wider population of doctors. Furthermore, few studies adjusted statistical analyses to account for factors (eg, workload) likely to impact the risk of malpractice claim or impaired performance. Finally, the research regarding characteristics and risk factors for malpractice and/or impaired performance predominantly comes from the USA. It is possible that differences in healthcare culture (eg, attitudes towards healthcare, spending and responsibility) and governance (eg, malpractice legislation) are country specific, thereby, limiting the generalisability of the review findings.

\section{CONCLUSION}

We identified 23 risk factors for doctors at risk of malpractice claims, complaint and/or impaired performance. The risk factors include demographic factors such as age and gender, as well as work-based characteristics such as clinical workload and the geographical location in which a doctor practices. No single factor was a strong predictor of risk of claims, complaint or impaired performance, therefore, a range of workplace and demographic information should be collected to establish risk profiles.

Twitter Elizabeth E Austin @DrLilAustin, Diana Fajardo Pulido @DFajardoPulido, Peter D Hibbert @peter_hibbert, Jeffrey Braithwaite @JBraithwaite1, Louise K Wiles @wileslouise79, Reidar P Lystad @RLystad, Janet C Long @JanetCLong, Frances Rapport @frapport and Robyn Clay-Williams @RClaywilliams

Acknowledgements Mary Simons contributed to the development of the search strategy and Catalin Tufanaru provided guidance on the selection of risk of bias tools for critical appraisal.

Contributors EEA, VD, RN, DFP, AP and RC-W conceived the review, RN conducted the search. EEA, VD, RN, DFP, RC-W, GA, YT, RPL, TT, SH, JCL and FR screened titles and abstracts. EEA, VD, RN, DFP, RC-W, GA, PDH, LKW and TT screened full texts. $\mathrm{EEA}, \mathrm{VD}, \mathrm{RN}, \mathrm{DFP}$ and RC-W extracted data and undertook the critical appraisal. $\mathrm{EEA}, \mathrm{VD}, \mathrm{DFP}$ and RC-W undertook the qualitative analysis. EEA and VD wrote the introduction, EA and RC-W wrote the discussion. RC-W, PDH and JB revised the first draft of the paper. All members of the team revised the final draft paper.

Funding This work was supported by The Medical Council of New South Wales. Competing interests None declared.

Patient consent for publication Not required.

Provenance and peer review Not commissioned; externally peer reviewed. 
Data availability statement All data relevant to the study are included in the article or uploaded as online supplemental information. All data relevant to the study are included in the article.

Supplemental material This content has been supplied by the author(s). It has not been vetted by BMJ Publishing Group Limited (BMJ) and may not have been peer-reviewed. Any opinions or recommendations discussed are solely those of the author(s) and are not endorsed by BMJ. BMJ disclaims all liability and responsibility arising from any reliance placed on the content. Where the content includes any translated material, BMJ does not warrant the accuracy and reliability of the translations (including but not limited to local regulations, clinical guidelines, terminology, drug names and drug dosages), and is not responsible for any error and/or omissions arising from translation and adaptation or otherwise.

Open access This is an open access article distributed in accordance with the Creative Commons Attribution Non Commercial (CC BY-NC 4.0) license, which permits others to distribute, remix, adapt, build upon this work non-commercially, and license their derivative works on different terms, provided the original work is properly cited, appropriate credit is given, any changes made indicated, and the use is non-commercial. See: http://creativecommons.org/licenses/by-nc/4.0/.

\section{ORCID iDs}

Elizabeth E Austin http://orcid.org/0000-0002-8438-2362

Peter D Hibbert http://orcid.org/0000-0001-7865-343X

Jeffrey Braithwaite http://orcid.org/0000-0003-0296-4957

Gaston Arnolda http://orcid.org/0000-0003-4948-7633

Reidar P Lystad http://orcid.org/0000-0003-0506-0902

Janet C Long http://orcid.org/0000-0002-0553-682X

Frances Rapport http://orcid.org/0000-0002-4428-2826

Robyn Clay-Williams http://orcid.org/0000-0002-6107-7445

\section{REFERENCES}

1 Krupat E, Dienstag JL, Padrino SL, et al. Do professionalism Lapses in medical school predict problems in residency and clinical practice? Acad Med 2020;95:888-95. doi:10.1097/ ACM.0000000000003145

2 Merriam-Webster. Impairment [online]. Available: https://www merriam-webster.com/dictionary/impairment [Accessed $15 \mathrm{Jul}$ 2020].

3 Kornmehl H, Singh S, Adler BL, et al. Characteristics of medical liability claims against dermatologists from 1991 through 2015. JAMA Dermatol 2018;154:160. doi:10.1001/jamadermatol.2017.3713

4 Otaki Y, Ishida MD, Saito Y, et al. Analysis of closed claims in the clinical management of rheumatoid arthritis in Japan. Chin Med $J$ 2017;130:1454-8.

5 Spittal MJ, Bismark MM, Studdert DM. Identification of practitioners at high risk of complaints to health profession regulators. BMC Health Serv Res 2019;19:380.

6 Nowotny BM, Loh R, Lorenz K. Sharing the pain: lessons from missed opportunities for healthcare improvement from patient complaints and litigation in the Australian health system. Australian Health Rev 2018;43.

7 Bismark MM, Spittal MJ, Gurrin LC, et al. Identification of doctors at risk of recurrent complaints: a national study of healthcare complaints in Australia. BMJ Qual Saf 2013;22:532-40.

8 Taylor N, Clay-Williams R, Hogden E, et al. High performing hospitals: a qualitative systematic review of associated factors and practical strategies for improvement. BMC Health Serv Res 2015;15.

9 Spittal MJ, Bismark MM, Studdert DM. The PRONE score: an algorithm for predicting doctors' risks of formal patient complaints using routinely collected administrative data. BMJ Qual Saf 2015;24:360-8. doi:10.1136/bmjqs-2014-003834

10 Moher D, Liberati A, Tetzlaff J, et al. Preferred reporting items for systematic reviews and meta-analyses: the PRISMA statement. PLoS Med 2009;6:e1000097.

11 Ouzzani M, Hammady H, Fedorowicz Z, et al. Rayyan-a web and mobile APP for systematic reviews. Syst Rev 2016;5:210. doi:10.1186/s13643-016-0384-4

12 Tufanaru C, Munn Z, Aromataris E. Chapter 3: Systematic reviews of effectiveness. In: Aromataris EMZ, ed. Joanna Briggs institute reviewer's manual. 2017. The Joanna Briggs Institute, 2017. https:// joannabriggs.org/ebp/critical_appraisal tools

13 Moola S, Munn Z, Tufanaru C. Chapter 7: Systematic reviews of etiology and risk. In: Aromataris E, Munn Z, eds. Joanna Briggs Institute reviewer's manual. The Joanna Briggs Institute, 2017. https://joannabriggs.org/ebp/critical_appraisal_tools
14 Fathy CA, Pichert JW, Domenico H, et al. Association between ophthalmologist age and unsolicited patient complaints. JAMA Ophthalmol 2018;136:61. doi:10.1001/jamaophthalmol.2017.5154

15 Nikoghosyan-Bossen G, Hauberg A, Homøe P. Increased number of ear-nose-throat malpractice complaints in Denmark. Dan Med J 2012;59:A4321.

16 Alam A, Klemensberg J, Griesman J. The characteristics of physicians disciplined by professional colleges in Canada. Open Med 2011;5:166-72.

17 Azab SMS. Claims of malpractice investigated by the Committee of medical ethics, Egyptian medical Syndicate, Cairo. Egyptian Journal of Forensic Sciences 2013;3:104-11.

18 Gómez-Durán EL, Martin-Fumadó C, Benet-Travé J, et al. Malpractice risk at the physician level: Claim-prone physicians. $J$ Forensic Leg Med 2018;58:152-4.

19 Schaffer AC, Babayan A, Yu-Moe CW, et al. The effect of clinical volume on annual and per-patient encounter medical malpractice claims risk. J Patient Saf 2020 doi:10.1097/ PTS.0000000000000706

20 Makowski MS, Shanafelt TD, Hausel A. Associations between dietary patterns and sleep-related impairment in a cohort of community physicians: a cross-sectional study. Am J Lifestyle Med 2019;55.

21 Cooper WO, Martinez W, Domenico HJ, et al. Unsolicited patient complaints identify physicians with evidence of neurocognitive disorders. Am J Geriatr Psychiatry 2018;26:927-36.

22 Del Bene VA, Brandt J. Identifying neuropsychologically impaired physicians. Clin Neuropsychol 2020;34:318-31. doi:10.1080/138540 46.2019.1666922

23 Brooks E, Gendel MH, Parry AL, et al. Challenging cognitive cases among physician populations: case vignettes and recommendations. Occup Med 2017;67:68-70. doi:10.1093/occmed/kqw132

24 Salmoirago-Blotcher E, Fitchett G, Leung K, et al. An exploration of the role of religion/spirituality in the promotion of physicians' wellbeing in emergency medicine. Prev Med Rep 2016;3:189-95.

25 Brooks E, Gendel MH, Early SR, et al. Physician boundary violations in a physician's health program: a 19-year review. J Am Acad Psychiatry Law 2012;40:59-66.

26 Buhl A, Oreskovich MR, Meredith CW, et al. Prognosis for the recovery of surgeons from chemical dependency: a 5-year outcome study. Arch Surg 2011;146:1286-91. doi:10.1001/archsurg.2011.271

27 Wile $\mathrm{C}$, Frei M, Jenkins K. Doctors and medical students case managed by an Australian doctors health program: characteristics and outcomes. Australas Psychiatry 2011;19:202-5. doi:10.3109/103 98562.2011 .561846

28 Finlayson AJR, Dietrich MS, Neufeld R, et al. Restoring professionalism: the physician fitness-for-duty evaluation. Gen Hosp Psychiatry 2013;35:659-63

29 Oreskovich MR, Shanafelt T, Dyrbye LN, et al. The prevalence of substance use disorders in American physicians. Am J Addict 2015;24:30-8. doi:10.1111/ajad.12173

30 Oreskovich MR, Kaups KL, Balch CM, et al. Prevalence of alcohol use disorders among American surgeons. Arch Surg 2012;147:168. doi:10.1001/archsurg.2011.1481

31 Elkin KJ, Spittal MJ, Elkin DJ. Doctors disciplined for professional misconduct in Australia and New Zealand, 2000-2009. Med J Australia 2011

32 Tibble HM, Broughton NS, Studdert DM. Why do surgeons receive more complaints than their physician peers? ANZ J Surg 2018;88:269-73.

33 Brooks E, Gendel M, Gundersen D. Physician health programmes and malpractice claims: reducing risk through monitoring. Occup Med 2013.

34 Studdert DM, Spittal MJ, Zhang Y. Changes in practice among physicians with malpractice claims. N Engl J Med Overseas Ed 2019

35 Millbank J. Health practitioner regulation: has the National law produced national outcomes in serious disciplinary matters? Federal Law Review 2019;47:631-54. doi:10.1177/0067205X19875028

36 Baker SR, Whang JS, Luk L, et al. The demography of medical malpractice suits against radiologists. Radiology 2013;266:539-47. doi:10.1148/radiol.12110971

37 Brooks SK, Chalder T, Gerada C. Doctors vulnerable to psychological distress and addictions: treatment from the practitioner health programme. J Ment Health 2011;20:157-64. doi:10.3109/09638237.2 011.556168

38 Walton M, Kelly PJ, Chiarella EM, et al. Profile of the most common complaints for five health professions in Australia. Aust Health Rev 2020;44:15. doi:10.1071/AH18074

39 Kynes JM, Schildcrout JS, Hickson GB, et al. An analysis of risk factors for patient complaints about ambulatory anesthesiology care. Anesth Analg 2013;116:1325-32. doi:10.1213/ ANE.0b013e31827aef83 
40 American Medical Association D of S and DR. Physician characteristics and distribution in the US, 2011: 1-46.

41 Qureshi HA, Rawlani R, Mioton LM, et al. Burnout phenomenon in U.S. plastic surgeons: risk factors and impact on quality of life. Plast Reconstr Surg 2015;135:619-26. doi:10.1097/ PRS. 000000000000855

42 Cottler LB, Ajinkya S, Merlo LJ, et al. Lifetime psychiatric and substance use disorders among impaired physicians in a physicians health program: comparison to a general treatment population: psychopathology of impaired physicians. J Addict Med 2013;7:108-12.

43 Yen W, Thakkar N. State of the science on risk and support factors to physician performance: a report from the Pan-Canadian physician factors collaboration. J Med Regul 2019;105:6-21 . doi:10.30770/2572-1852-105.1.6

44 Chen K-Y, Yang C-M, Lien C-H, et al. Burnout, job satisfaction, and medical malpractice among physicians. Int J Med Sci 2013;10:1471-8.

45 Liu J, Hyman DA. Targeting bad doctors: lessons from Indiana, 1975-2015. J Empir Leg Stud 2019;16:248-80. doi:10.1111/ jels. 12214

46 Huizinga $\mathrm{CRH}$, de Kam ML, Stockmann HBAC, et al. Evaluating fitness to perform in surgical residents after night shifts and alcoho intoxication: the development of a "fit-to-perform" test. J Surg Educ 2018;75:968-77.

47 St Hilaire MA, Anderson C, Anwar J, et al. Brief ( $<4 \mathrm{hr}$ ) sleep episodes are insufficient for restoring performance in first-year resident physicians working overnight extended-duration work shifts. Sleep 2019;42 doi:10.1093/sleep/zsz041

48 Tseng YW, Vedula SS, Malpani A. Association between surgical trainee daytime sleepiness and intraoperative technical skill when performing septoplasty. JAMA Facial Plast Surg 2019;21:104-9.

49 Anderson C, Sullivan JP, Flynn-Evans EE, et al. Deterioration of neurobehavioral performance in resident physicians during repeated exposure to extended duration work shifts. Sleep 2012;35:1137-46. doi:10.5665/sleep.2004

50 Wilson M, Randhawa H, Lavis J. Rapid synthesis: identifying risk and protective factors for quality clinical practice [online]. Hamilton, Canada, 2015https://macsphere.mcmaster.ca/handle/11375/17255 [Accessed 7 Aug 2020]

51 Bismark MM, Spittal MJ, Studdert DM. Prevalence and characteristics of complaint-prone doctors in private practice in Victoria. Med J Aust 2011;195:25-8.

52 Studdert DM, Bismark MM, Mello MM, et al. Prevalence and characteristics of physicians prone to malpractice claims. N Engl J Med 2016;374:354-62. doi:10.1056/NEJMsa1506137

53 Elkin K, Spittal MJ, Elkin D, et al. Removal of doctors from practice for professional misconduct in Australia and New Zealand. BMJ Qual Saf 2012;21:1027-33. doi:10.1136/bmjqs-2012-000941

54 Balch CM, Oreskovich MR, Dyrbye LN, et al. Personal consequences of malpractice lawsuits on American surgeons. J Am Coll Surg 2011;213:657-67.

55 Myers LC, Gartland RM, Skillings J, et al. An examination of medical malpractice claims involving physician trainees. Acad Med 2020;95:1215-22. doi:10.1097/ACM.0000000000003117

56 Australian Institute of Health and Welfare. Medical practitioners workforce 2015 [online], 2016. Available: https://www.aihw.gov. au/getmedia/355dbfe6-9944-4dcf-98a2-472ca076458b/Medicalpractitioners-workforce-2015.pdf.aspx?inline=true

57 Australian Institute of Health and Welfare. Australia's medical indemnity claims 2012-13 [online]. Canberra, 2014. Available: https:// www.aihw.gov.au/reports/hospitals/medical-indemnity-claims-201213/contents/summary [Accessed 7 Aug 2020].

58 Jones AT, Kopp JP, Malangoni MA. Association between maintaining certification in general surgery and loss-of-license actions. JAMA 2018;320:1195. doi:10.1001/jama.2018.9550

59 Birkeland S, Bogh SB. General practice location and malpractice litigation. Rural Remote Health 2019;19:4663. doi:10.22605/ $\mathrm{RRH} 4663$

60 Carlson JN, Foster KM, Pines JM, et al. Provider and practice factors associated with emergency physicians' being named in a malpractice claim. Ann Emerg Med 2018;71:157-64.

61 Dallal RM, Pang J, Soriano I, et al. Bariatric-related medical malpractice experience: survey results among ASMBS members. Surg Obes Relat Dis 2014;10:121-4.
62 Tiffin PA, Paton LW, Mwandigha LM, et al. Predicting fitness to practise events in international medical graduates who registered as UK doctors via the professional and linguistic assessments board (PLAB) system: a national cohort study. BMC Med 2017;15:66. doi:10.1186/s12916-017-0829-1

63 Boyll P, Kang P, Mahabir R, et al. Variables that impact medical malpractice claims involving plastic surgeons in the United States. Aesthet Surg J 2018;38:785-92. doi:10.1093/asj/sjx182

64 Carney PA, Frederick PD, Reisch LM, et al. How concerns and experiences with medical malpractice affect dermatopathologists' perceptions of their diagnostic practices when interpreting cutaneous melanocytic lesions. J Am Acad Dermatol 2016;74:317-24.

65 Finkelstein J, Zhu S. Phenotyping physicians with frequent malpractice claims. In: Proceedings - 2017 IEEE International Conference on Bioinformatics and Biomedicine, BIBM 2017. Institute of Electrical and Electronics Engineers Inc. 2017:1567-70.

66 Lyu S-Y, Liao C-K, Chang K-P. Analysis of medical litigation among patients with medical disputes in cosmetic surgery in Taiwan. Aesthetic Plast Surg 2011;35:764-72.

67 AbuDagga A, Wolfe SM, Carome M, et al. Cross-Sectional analysis of the 1039 U.S. physicians reported to the National practitioner data bank for sexual misconduct, 2003-2013. PLoS One 2016;11:e0147800. doi:10.1371/journal.pone.0147800

68 Jena AB, Schoemaker L, Bhattacharya J, et al. Physician spending and subsequent risk of malpractice claims: observational study. BMJ 2015;351:h5516. doi:10.1136/bmj.h5516

69 Tsugawa Y, Jha AK, Newhouse JP, et al. Variation in physician spending and association with patient outcomes. JAMA Intern Med 2017:177:675. doi:10.1001/jamainternmed.2017.0059

70 Black B, Hyman DA, Lerner JY. Physicians with multiple paid medical malpractice claims: are they outliers or just unlucky? Int Rev Law Econ 2019;58:146-57.

71 Raineri Bernain G, Bravo Rodríguez L, Lagos Tissie D, et al. Analysis of Chilean Supreme Court rulings in medical malpractice cases, 2014-2015. Spanish Journal of Legal Medicine 2019;45:108-13.

72 Casali MB, Blandino A, Del Sordo S, et al. Alleged malpractice in orthopaedics. Analysis of a series of medmal insurance claims. $J$ Orthop Traumatol 2018;19:7. doi:10.1186/s10195-018-0500-4

73 Faisant M, Papin-Lefebvre F, Rerolle C, et al. Twenty-five years of French jurisprudence in criminal medical liability. Med Sci Law 2018;58:39-46. doi:10.1177/0025802417737402

74 Hamasaki T, Hagihara A. Physicians' explanatory behaviours and legal liability in decided medical malpractice litigation cases in Japan. BMC Med Ethics 2011;12:7. doi:10.1186/1472-6939-12-7

75 Bishop TF, Ryan AM, Ryan AK, et al. Paid malpractice claims for adverse events in inpatient and outpatient settings. JAMA 2011;305:2427-31. doi:10.1001/jama.2011.813

76 Gaitan-Duarte H, Eslava-Schmalbach J, Montoya L, et al. Association between reportable preventable adverse events and unfavorable decisions in medical malpractice claims involving obstetricians covered by FEPASDE Colombia 1999 to 2014. Casecontrol study. Colomb J Anesthesiol 2019;47:14-22. doi:10.1097/ CJ9.0000000000000081

77 Mozeika AM, Sachdev D, Asri R, et al. Sociological and medical factors influence outcomes in facial trauma malpractice. J Oral Maxillofac Surg 2019;77:1042.e1-1042.e10.

78 Medical Board AHPRA. Registration data table - March 2020 [online] 2020. Available: https://www.medicalboard.gov.au/News/Statistics. aspx

79 Brooks E, Early SR, Gundersen DC, et al. Comparing substance use monitoring and treatment variations among physician health programs. Am J Addict 2012;21:327-34. doi:10.1111/j.15210391.2012.00239.x

80 Keshavarz P, Dehghani M, Malekpour F, et al. A 10-year retrospective descriptive study of internists' complaints referred to Fars Legal Medicine Center. Egypt J Forensic Sci 2019;9. doi:10.1186/s41935019-0130-X

81 Oshel RE, Levitt P. The detection, analysis, and significance of physician clustering in medical malpractice lawsuit Payouts. J Patient Saf 2016:1-5.

82 Tessler MJ, Shrier I, Steele RJ. Association between anesthesiologist age and litigation. Anesthesiology 2012;116:574-9.

83 Yadav M, Rastogi P. A study of medical negligence cases decided by the district consumer courts of Delhi. $J$ Ind Acad Foren Med 2015;37:50-5. 